\title{
Syria
}

Archéologie, art et histoire

95 | 2018

Dossier : Sur les routes de Syrie et d'Asie Mineure

\section{Les fortifications hellénistiques de Cyrrhus-Nebi Houri : étude archéologique et géophysique}

Mathilde Gelin

\section{(2) OpenEdition}

\section{Journals}

Édition électronique

URL : http://journals.openedition.org/syria/7701

DOI : 10.4000/syria.7701

ISSN : 2076-8435

Éditeur

IFPO - Institut français du Proche-Orient

\section{Édition imprimée}

Date de publication : 31 décembre 2018

Pagination : 375-395

ISBN : 978-2-35159-750-7

ISSN : 0039-7946

\section{Référence électronique}

Mathilde Gelin, « Les fortifications hellénistiques de Cyrrhus-Nebi Houri : étude archéologique et géophysique », Syria [En ligne], 95 | 2018, mis en ligne le 01 mai 2021, consulté le 03 mai 2021. URL: http://journals.openedition.org/syria/7701 ; DOI : https://doi.org/10.4000/syria.7701 


\title{
SYRIA ARCHÉOLOGIE, ART ET HISTOIRE
}

\author{
revue publiée par \\ l'Institut français du Proche-Orient
}

Tome 95

Année 2018

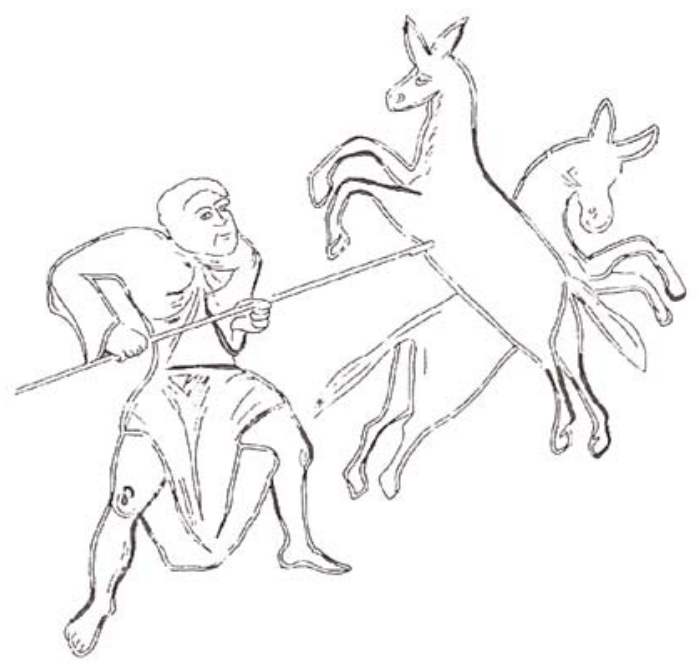

avec le concours du Ministère de l'Europe et des Affaires Étrangères (MEAÉ-DGM) et du Centre national de la recherche scientifique (UMIFRE 6, USR 3135)

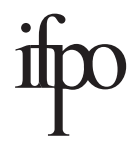

BEYROUTH 
Vignette de couverture : Détail d'une peinture murale de Qusayr 'Amra

(relevé C. Vibert-Guigue \& Gh. Bisheh, Les peintures de Qusayr 'Amra [BAH 179], Beyrouth 2007). 
Syria est une revue périodique annuelle publiée par l'Institut français du Proche-Orient

(UMIFRE 6, CNRS-MEAÉ, USR 3135).

\section{DIRECTION}

Éditeur :

Michel Mouton, directeur de l'Ifpo

Directeurs de la revue :

Françoise BRIQuel ChatonNEt, directrice de recherche au CNRS

Rédacteur en chef :

Dominique PIERI, directeur scientifique pour l'archéologie et l'histoire de l'Antiquité à l'Ifpo Françoise BRIQuel ChATONNET

\section{SECRÉTARIAT DE RÉDACTION}

Le secrétariat de rédaction de Syria, assuré par Gaëlle CoQUEUGNIOT (courriel : syria @ cnrs.fr), est placé sous la responsabilité du rédacteur en chef de la revue. Les articles et communications scientifiques, les ouvrages dont on désire un compte rendu, et toute la correspondance afférente doivent êtres adressés à Gaëlle Coqueugniot, secrétariat de rédaction de Syria, Maison de l'Archéologie et de l'Ethnologie René-Ginouvès, 21, allée de l’Université, 92023 - Nanterre Cedex, France.

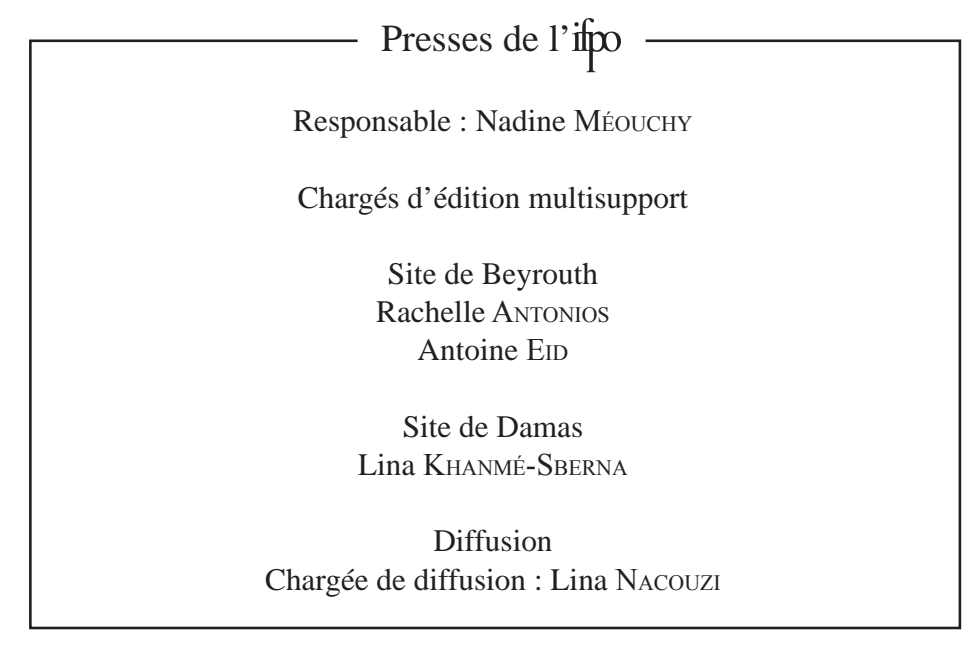

(C) 2018, Presses de l'Ifpo

B.P. 11-1424 Beyrouth, Liban

Tél./Fax : + $961(0) 1420294$

www.ifporient.org

Courriel : diffusion@ifporient.org

SYRIA en ligne :

http://journals.openedition.org/syria

http://www.persee.fr/web/revues/home/prescript/revue/syria

http://www.jstor.org/action/showPublication?journalCode=syria

ISSN 0039-7946

ISBN 978-2-35159-750-7

Dépôt légal : $2^{\mathrm{e}}$ trimestre 2019 


\section{COMITÉ DE RÉDACTION}

Syria est administrée par un comité de rédaction incluant l'éditeur et les deux directeurs de la revue, entourés de :

Frédéric AlPI, ingénieur de recherche, CNRS, Ifpo, Beyrouth

Pascal Butterlin, professeur, Université Paris 1 Panthéon-Sorbonne

Denis Genequand, Université de Genève

Philippe QuENET, professeur, Université de Strasbourg

Carole Roche-Hawley, directeur de recherche, CNRS, Paris

Laurent THOLBECQ, professeur, Université libre de Bruxelles

François Villeneuve, professeur, Université Paris 1 Panthéon-Sorbonne

Jean-Baptiste Yon, directeur de recherche, CNRS, Lyon-Beyrouth

Ce comité officie également pour les ouvrages de la collection BAH (Bibliothèque archéologique et historique) de l'Ifpo.

\section{COMITÉ DE LECTURE}

\begin{tabular}{|c|c|c|c|c|c|}
\hline$F_{R A N C E}$ & ALLEMAGNE & ESPAGNE & JORDANIE & POLOGNE & RUSSIE \\
\hline R. BOUCHARLAT & H. KÜHNE & A. Almagro & Gh. BISHEH & M. GAWLIKOWSKI & G. Koshelenko \\
\hline A. CAubet & W. ORTHMANN & M. Molist & F. ZAYADINE & & \\
\hline D. ChARPIN & T. Ulbert & & & ROYAUME- $U_{N I}$ & SUISSE \\
\hline H. DE Contenson & T. WeBER & $\dot{E}_{T A T S}-U_{N I S}$ & LIBAN & M. MACDONALD & C. BONNET \\
\hline J.-M. DentZER & & G. W. Bowersock & L. BADRE & C. MANGO & R. StUCKу \\
\hline D. FeISSEL & BELGIQUE & D. PARDEE & H. SALAMÉ-SARKIS & E. Peltenburg & \\
\hline J.-B. HUMBERT & J. BALTY & & & & SYRIE \\
\hline J.-L. Нuот & J.-Ch. BALtY & ITALIE & & & M. AL-MAQDISSI \\
\hline J.-C. MARGUERON & F. de Callata & A. BARONI & & & S. MuHEISEN \\
\hline J.-P. SodINI & & A. INVERNIZZI & & & \\
\hline M. YoN & DANEMARK & P. MatThiAe & & & \\
\hline & J. LuND & S. RIBICHINI & & & \\
\hline
\end{tabular}

\section{RECOMMANDATIONS AUX AUTEURS/RECOMMENDATIONS TO AUTHORS}

Syria publie des articles en français, en anglais, en allemand, en italien, en espagnol.

Chaque article doit être accompagné d'un bref résumé d'une dizaine de lignes en français et en anglais (un résumé en arabe est ensuite réalisé et ajouté par l'éditeur). L'ensemble, article et notes, ne doit pas excéder 25 pages imprimées (sauf accord particulier préalable). Les articles reçus sont soumis sans délai à l'avis de deux rapporteurs désignés par le rédacteur en chef. Dès réception de leur rapport, les auteurs sont avisés de l'acceptation ou du refus de leur article, ou de demandes de modifications éventuelles.

D’une façon générale, la charte éditoriale, portant des recommandations plus complètes concernant la présentation des manuscrits, peut être téléchargée sur le site web de l'Ifpo/Presses de l'Ifpo : http://www.ifporient.org/node/87.

Tous les articles proposés pour publication dans Syria doivent être envoyés sous forme imprimée et numérique à Gaëlle Coqueugniot : syria@cnrs.fr, secrétaire de rédaction, et sous forme numérique uniquement à Françoise Briquel Chatonnet : francoise.briquel-chatonnet@cnrs.fr.

Syria publishes articles in French, English, German, Italian, Spanish.

Every submission should include a short abstract (10 lines) in French and English. An Arabic abstract is then written and added by the publisher. An article will not exceed 25 printed pages (including footnotes) unless agreed otherwise. Every article received is promptly submitted to two referees designated by the editor-in-chief. On receipt of their report, authors are informed whether their article has been accepted for publication or not, or are notified of any modification that may be required.

More generally, an editorial guide with more complete guidelines about the display of articles, can be downloaded from the website of Ifpo/ Presses de l'Ifpo/Charte éditorale: http://www.ifporient.org/node/87.

All articles submitted to Syria should be sent in hard copy and digital version to the assistant editor Gaëlle Coqueugniot: syria@cnrs.fr and in digital version only to Françoise Briquel Chatonnet: francoise.briquel-chatonnet@cnrs.fr. 


\section{SYRIA, TOME 95, ANNÉE 2018}

\section{SOMMAIRE}

ISSN 0039-7946

ISBN 978-2-35159-750-7

Éditorial

I - Dossier : Sur les routes de Syrie et d’Asie Mineure - Hommage à Maurice Sartre (coordonné par Catherine Grandjean)

Grandjean (C.), Avant-propos

BaLty (J.-C.), Cippes, autels funéraires et stèles militaires d'Apamée : typologie des monuments, modèles iconographiques et ateliers

Bowersock (G. W.), Religion in Early Fourth Century Roman Palestine: The Kfar 'Othnay Mosaics

GATIER (P.-L.), Le chameau et l'onagre, viandes des Ismaélites.

Mitchell (S.), L'alimentation en Asie Mineure à travers les siècles. Esquisse d'une histoire culturelle ....111

Aliquot (J.), Regio Parhalia. Genèse et administration d'un district domanial de la Syrie romaine

Boulay (T.), La liberté de Téos et le soutien d'Attale II à Alexandre Balas 133

Bader (N.) et Yon (J.-B.), Une inscription du théâtre de Bayt Ras/Capitolias 155

$$
\text { II - Autres Articles }
$$

Poupet (P.), Baldi (J. S.) et Harfouche (R.),

Matériaux pour céramiques à l'âge du Bronze, dans la vallée du Nahr Ibrahim (Liban)............171

AlachKar (S.), Euphrates Banded Wares et leur contexte funéraire. Un nouveau bilan .............................197

Blétry (S.), Duval (H.), Girardi (C.), Loupmon (T.) et Rjoob (A.),

Tell Keila, résultats de quatre années de recherches.

Mavrojannis (T.), Le commerce des esclaves syriens (143-88 av. J.-C.) .....

KuBiak-Schneider (A.), Une inscription palmyrénienne inédite à " Miséricordieux »....

Hutton (J. M.), Kumon (H.), McLaughlin (M.) et Atwood (P. L.), Two Palmyrene funerary

busts in the collection of the Nelson-Atkins Museum of Art, Kansas City, Mo

Kaizer (T.) et Raja (R.), Divine symbolism on the tesserae from Palmyra.

Considerations about the so-called "Symbol of Bel" or "Signe de la pluie"

BARAZE (M.), Un ensemble funéraire (IV - IX siècle) découvert dans le marché suburbain de Palmyre......317

Ahmad (T.), La fontana monumentale del santuario di Damasco - Siria (Sūq l-ṣāga) .....

Claes (L.) et Tavernier (J.), Exit Gordianus, but how? Shapur's trilingual inscription revisited 
GeLIN (M.), Les fortifications hellénistiques de Cyrrhus-Nebi Houri :

étude archéologique et géophysique.

Abdul Massin (J.), Développement et urbanisme du secteur méridional de Cyrrhus-Nebi Houri

Al-ShBiB (Sh.), La défense des villes à l'époque byzantine : alternance entre les tours et les bastions, tradition ou innovation?

$$
\text { III - VARIÉTÉS }
$$

Angevin (R.), Les industries lithiques de Tell Chuera et Kharab Sayyar (Syrie du Nord):

nouvelles perspectives techno-économiques et implications historiques

Lempereur (O.) et Aliquot (J.), Une stèle funéraire d'Antioche au musée archéologique de Viuz-Faverges (Haute-Savoie)

SARTRE (M.), Retour vers la Coelé-Syrie

$$
\text { IV - NÉCROLOGIES }
$$

Ali Abou Assaf, la beauté morale des valeurs archéologiques

('Atil 23 septembre 1931-'Atil $1^{\text {er }}$ mars 2018) [M. AL-MAQDISSI] 463

Javier Teixidor (13 novembre 1930-25 septembre 2017) [F. BRIQuel ChatonNET]

$$
\mathrm{V}-\text { ReCENSIONS }
$$

Les recensions sont désormais publiées exclusivement en ligne, sur le site https://journals.openedition.org/syria/

Les ouvrages suivants ont fait l'objet d'une recension dans l'année 2018 :

Nicolo Marchetti, Kinku, sigilli dell'eta del Bronzo dalla Regione di Gaziantep in Turchia (Orientlab 1), Bologne, Ante quem, 2011 [Pascal ButTerLin]

Felix Höflmayer et Ricardo Eichmann (éd.), Egypt and the Southern Levant in the Early Bronze Age (OrientArchäologie 31), Rahden/Westf., Leidorf, 2014 [Christophe NiCOLLE]

Nicolò Marchetti (éd.), Karkemish. An Ancient Capital on the Euphrates, Bologne, Ante Quem, 2014 [Christine KEPINSKI]

D. Praet et B. Bakhouche (éd.), Franz Cumont. Astrologie (Bibliotheca Cumontiana. Scripta Minora IV), Turnhout, 2014 [Jean LEMPIRE]

Mhamed Hassine Fantar, Maurice Sznycer, François Bron, Stèles à inscriptions néopuniques de Maktar, vol. 1 (Corpus des antiquités phéniciennes et puniques, France 3 / Tunisie 1, Mémoires de l'Académie des Inscriptions et Belles-Lettres 51), Paris, Diffusion De Boccard, 2015 [Jimmy DACCACHE]

Klaus Stefan Freyberger, Christine Ertel $\dagger$, Kathrin Tacke, Hassan Hatoum, Kanatha von hellenistischer bis spätantiker Zeit. Band I : Die Heiligtümer. Orte der Herrschaft und urbane Kommunikationszentren (Damaszener Forschungen 16), Mayence, Philipp von Zabern, 2015 [Maurice SARTRE] 
Karel J. H. Vriezen et Ute Wagner-Lux (éd.), Gadara-Umm Qès II. The Twin Churches on the RomanByzantine Terrace and the Excavations in the streets (Abhandlungen des Deutschen Palästina-Vereins 30/2), Wiesbaden, Harrassowitz, 2015 [Bertrand RIBA]

Zbigniew T. Fiema, Jaakko Frösén et Maija Holappa (éd.), Petra - The Mountain of Aaron. The Finnish Archaeological Project in Jordan. II. The Nabataean Sanctuary and the Byzantine Monastery, Helsinki, Societas Scientiarum Fennica, 2016 [Maurice SARTRE]

Emmanuel Laroche, Études anatoliennes (Subartu 37), Turnhout, Brepols, 2016 [Alice Mouton]

Dominique Parayre (dir.), Le fleuve rebelle, Géographie historique du moyen Oronte d'Ebla à l'époque médiévale, Actes du colloque international tenu les 13 et 14 décembre 2012 à Nanterre (MAE) et à Paris (INHA) (Syria Supplément IV), Beyrouth, Presses de l'Ifpo, 2016 [Jean-Louis Huot]

Hasan Peker, Texts from Karkemish I. Luwian Hieroglyphic Inscriptions from the 2011-2015 Excavations (OrientLab Series Maior 1), Bologne, Ante quem, 2016 [Alice Mouton]

Philippe Quenet (dir.), Ana ziqquratim. Sur la piste de Babel, Strasbourg, Presses Universitaires de Strasbourg, 2016 [Cécile Michel]

Shigeo Yamada et Daisuke Shibata (éd.), Cultures and Societies in the Middle Euphrates and Habur Areas in the Second Millennium BC-I. Scribal Education and Scribal Traditions (Studia Chaburensia vol. 5), Wiesbaden, Harrassowitz Verlag, 2016 [Bertrand LAFONT]

Cédric BRÉLAZ (éd.), L'héritage grec des colonies romaines d'Orient. Interactions culturelles dans les provinces hellénophones de l'empire romain, Paris, De Boccard, 2017 [Maurice SARTRE]

Françoise Briquel Chatonnet et Muriel Debié, Le monde syriaque. Sur les routes d'un christianisme ignoré, Paris, Les Belles Lettres, 2017 [Sebastian BRock]

Ross Burns, Origins of the Colonnaded Streets in the Cities of the Roman East, Oxford/New York, Oxford University Press, 2017 [Catherine SALIOU]

Boris Chrubasik et Daniel KIng (éd.), Hellenism and the local communities of the Eastern Mediterranean, 400 BCE-250 CE, Oxford, Oxford University Press, 2017 [Maurice SARTRE]

Aaron Tugendhaft, Baal and the Politics of Poetry (The Ancient Word), New York, Routledge, 2018 [Stéphanie ANTHONIOZ] 


\title{
LES FORTIFICATIONS HELLÉNISTIQUES DE CYRRHUS-NEBI HOURI : ÉTUDE ARCHÉOLOGIQUE ET GÉOPHYSIQUE *
}

Résumé - La cité antique de Cyrrhus-Nebi Houri occupe une position stratégique privilégiée qui a marqué d'un caractère éminemment militaire sa destinée et, sur le terrain, ses fortifications encore bien conservées témoignent de ce passé. Les limites urbaines que ces dernières matérialisent ont été particulièrement étudiées par la mission syro-libanaise qui a pu constater que, contrairement aux autres parties de la ville, les murailles méridionales des époques romaine, byzantine et médiévale n'épousent pas le même tracé que celui de la période hellénistique. La recherche du tracé d'origine de ces fortifications a été menée dans un premier temps au moyen de la fouille archéologique ciblée, qui a permis de restituer l'image d'une ville de moins grandes dimensions mais s'adaptant au relief afin d'en exploiter toutes les possibilités offertes à la défense. Dans un deuxième temps l'étude géophysique, tout en confirmant les découvertes archéologiques, a permis de connaitre l'intégralité du tracé du rempart hellénistique et offre de nombreuses et nouvelles pistes de recherche et d'interprétation.

Mots-clés - Syrie, Cyrrhus, Nebi Houri, période hellénistique, stratégie de défense, architecture militaire, fortifications, limites urbaines.

\begin{abstract}
The ancient city of Cyrrhus-Nebi Huri occupies an ideal strategic position with a strong military characteristic and, on the field, its well preserved fortifications testify to this past. The urban boundaries that they materialize have been particularly studied by the Syro-Lebanese mission, which was able to observe that, unlike the other parts of the city, the southern walls of the Roman, Byzantine and Medieval periods do not follow the same line as that of the Hellenistic period. The search for the original line of these fortifications was initially carried out by focused archaeological excavation, which resulted in the image of a city of smaller dimensions but adapting to the relief, exploiting all the possibilities offered to the defense. In a second phase, the geophysical study, while confirming the archaeological discoveries, made it possible to uncover the entire course of the Hellenistic rampart and offers many new directions for further research.
\end{abstract}

Keywords - Syria, Cyrrhus, Nebi Huri, Hellenistic period, defense strategy, military architecture, fortifications, urban limits.

* Cette étude a pour origine une intervention donnée par l'auteure en juin 2011 à la table ronde organisée par C. Benech, CNRS (UMR 5133 Archéorient) et la Direction générale des antiquités et des musées de Syrie, afin de présenter les résultats de l'ANR Prospection géophysique et étude des centres urbains de Syrie antiquePROGECESA JC07-191982. Je remercie tout particulièrement l'ANR et C. Benech de m'avoir conviée à y participer. La rédaction de Syria remercie Chadi Hatoum (docteur en archéologie, UMR 7041 ArScAn) pour la traduction du résumé et des mots-clés en arabe. 


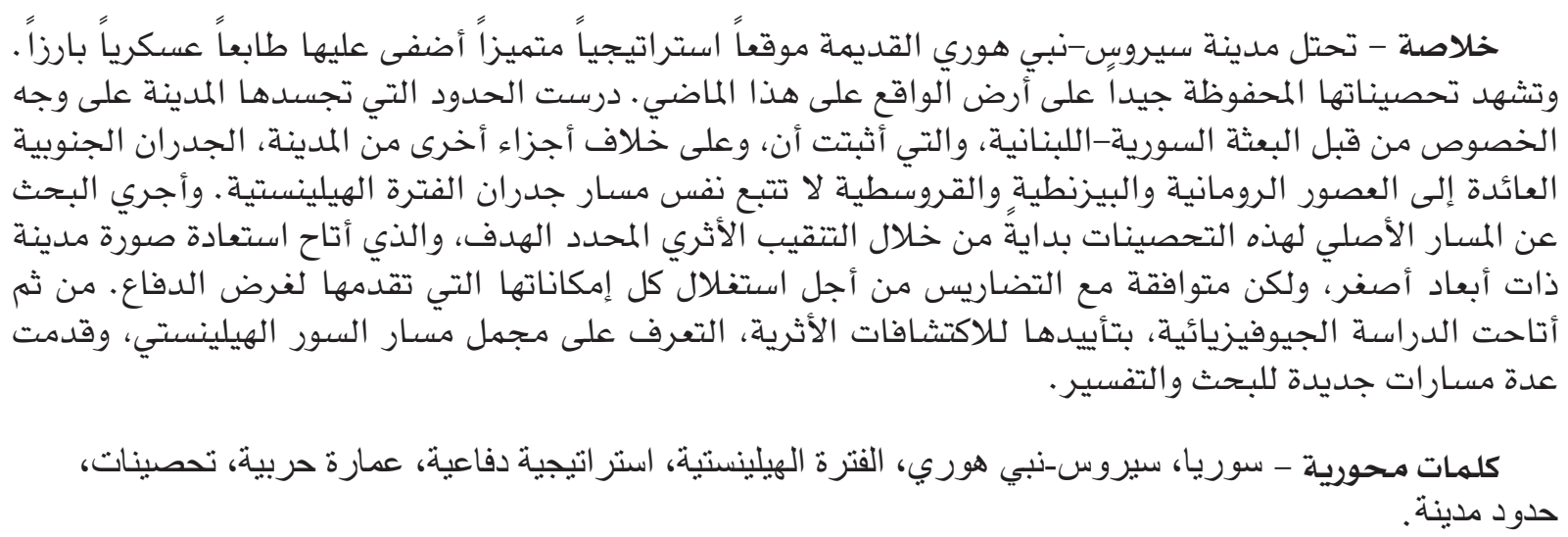

Cyrrhus-Nebi Houri se situe à l'extrême nord-ouest de la Syrie (région de Afrin), à moins de deux kilomètres de la frontière syro-turque. La fondation de la ville a été attribuée à l'époque hellénistique ${ }^{1}$ et associée à un programme de fondations urbaines ${ }^{2}$ tel celui que Séleucos ${ }^{\text {er }}$ mît en place, vers 300 av. J.-C. ${ }^{3}$, en dépit du fait que ni les campagnes de fouilles, ni les sources ne permettent de l'affirmer ${ }^{4}$. Par la suite, à partir de 64 av. J.-C., Cyrrhus a connu une occupation romaine, puis byzantine du IV ${ }^{\mathrm{e}}$ au VII ${ }^{\mathrm{e}}$ siècles, et arabe jusqu'au XII ${ }^{\mathrm{e}}$ siècle $^{5}$.

L'implantation de la ville, sur un relief dominant la région proche, en a fait une position stratégique privilégiée qui a marqué son histoire d'un caractère éminemment militaire. D'après les recherches antérieures ${ }^{6}$, à l'époque hellénistique Kyrrhos, capitale de la région de Cyrrhestique, placée sur les voies de circulation reliant les principales villes voisines, fut alors fortifiée et très probablement dotée d'une citadelle, haut lieu de la défense et de l'activité militaire (fig. 1). À la période romaine, elle devient un poste de défense face aux Parthes; les Romains interviennent alors sur les murailles en plusieurs endroits, allant jusqu'à bâtir de nouvelles parties, notamment dans le secteur méridional. Centre épiscopal et métropole à l'époque byzantine, elle voit la mise en place par Justinien d'un vaste programme de reconstruction des murailles. Enfin, devenue un poste militaire aux périodes islamiques, la ville connaît d'autres remaniements des fortifications, principalement à la citadelle.

Dès sa création en 2006 sous la tutelle scientifique de l'Université Libanaise (UL) et de la Direction générale des antiquités et des musées de Syrie (DGAMS), la mission syro-libanaise, dirigée par J. Abdul Massih et Sh. Al Shbib ${ }^{7}$, a défini comme l'un de ses thèmes de recherche principaux l'étude « de l'occupation territoriale de la ville ${ }^{8}$ et ses relations avec l'urbanisme, aux différentes époques de son existence. Dans le cadre de cette problématique, la recherche de la première ville hellénistique et de ses limites urbaines représentées par les fortifications a été conduite en priorité. Afin de mener à bien cette recherche dans les meilleurs délais et, étant données les dimensions des murailles et les différentes

1. Abdul Massih 2012, p. 303.

2. FrÉZOUls 1977, p. 179.

3. Abdul Massih 2012, p. 303.

4. La ville est mentionnée de manière indirecte, au cours d'événements survenus en 221 av. J.-C. : Polybe, Histoires, V, 50,7 et 57, 4, Frézouls 1977, p. 179 et FrÉzouls 2012, p. 81-87.

5. Pour les détails sur l'histoire de Cyrrhus, voir Abdul Massin 2012, Frézouls 1977, p. 178-189 et Frézouls 2012, p. 79-102.

6. Voir plus bas.

7. Respectivement professeure à l'UL et responsable de la Syrie du Nord à la DGAMS. Je les remercie tous deux pour m'avoir accordé leur confiance.

8. Abdul Massin 2012, p. 303. 


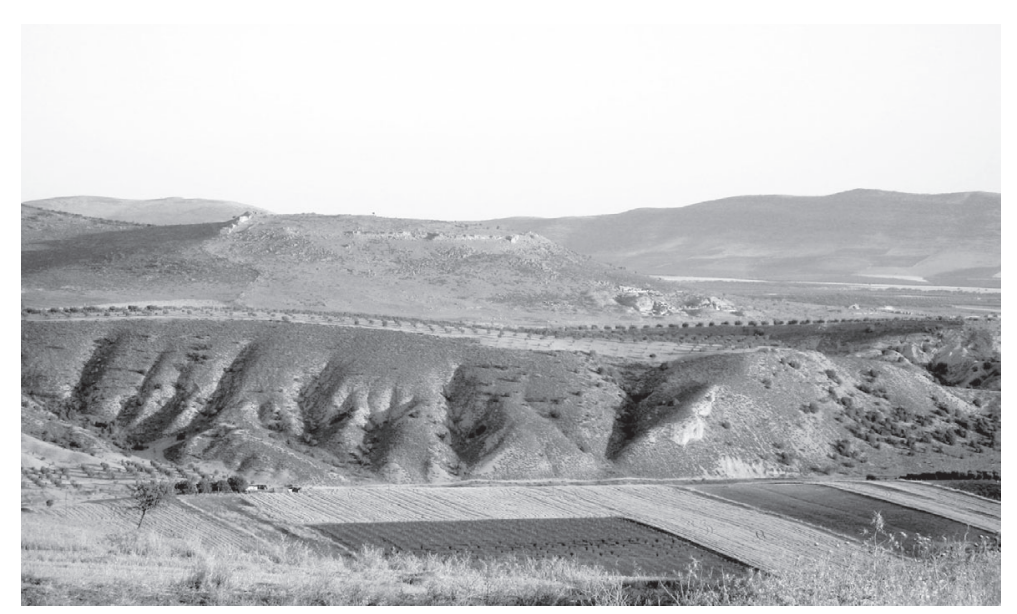

Figure 1. La citadelle dans ses dimensions actuelles, dominant la ville de Cyrrhus (vue vers l'ouest). Sur la gauche de l'image, on voit le tracé du rempart méridional qui descend vers la ville basse, formant plusieurs décrochements (C) M. Gelin.

études thématiques nécessaires à leur bonne compréhension, les études et les secteurs de fouille ont été répartis entre les différents chercheurs ${ }^{9}$.

La présente étude expose ma contribution à cette recherche sur les premières fortifications mises au jour à Cyrrhus et, plus précisément, sur une construction en polygonal qui se trouve sous les murailles méridionales de la citadelle et de la ville. Mon intervention s'est traduite, dans un premier temps par une investigation archéologique (fouilles et observations du terrain), dans un deuxième temps par l'examen de l'image issue de la prospection géophysique réalisée par C. Benech ${ }^{10}$.

9. Les recherches ont été réparties comme suit :

J. Abdul Massih a pris en charge la fouille des deux portes sud (de 2007 à 2011) et l'étude de l'implantation générale du plan d'urbanisme.

Sh. Al Shbib a étudié et fouillé plusieurs secteurs des remparts nord, ouest et est de la ville, ainsi que le rempart sud à l'exception de la porte et de la partie à l'ouest de la tour 5 (à partir d'un changement de direction de la muraille, voir fig. 9), de 2008 à 2011.

J.-C. Bessac a étudié une partie des fortifications et notamment les matériaux, les techniques et l'économie de la construction, ainsi que les possibilités de leur conservation (2006-2009).

C. Benech a procédé à la prospection géophysique de la ville basse méridionale (2009), puis à l'étude de la carte obtenue, selon une problématique centrée sur l'évolution de l'urbanisme de cette partie de la ville.

Il est à signaler que J. Humbert, qui a pris en charge les relevés des remparts (2008-2011) a pu, par sa connaissance des fortifications hellénistiques qu'il a observées sur d'autres sites, restituer certaines parties du tracé de la muraille de Cyrrhus (voir plus bas).

Enfin, je me suis concentrée sur l'étude de la citadelle (2007-2011), ainsi que sur la partie du rempart sud descendant de la citadelle vers la ville, jusqu'au-delà du changement de direction situé à l'ouest de la tour 5 (en 2008 ; voir fig. 9).

L'ensemble de ces recherches confère une portée collective aux résultats dont bénéficie la mission. Néanmoins, si les chercheurs ont procédé à de nombreux échanges entre eux, autorisant notamment les autres membres de l'équipe à utiliser leurs résultats, chaque étude a été menée individuellement, selon les thèmes de recherche propres à chacun. De la sorte, afin de ne pas risquer d'égarer le lecteur, j'emploierai la première personne du singulier pour mentionner mes recherches propres, et « la mission » lorsqu'il s'agit des recherches d'autres chercheurs ou « collectives », que plusieurs membres de la mission ont menées et auxquelles j'étais associée. Je remercie l'ensemble des membres de la mission de Cyrrhus pour leur sens du travail collectif.

10. Les fortifications visibles sur cette carte n'entrant pas dans la thématique de recherche alors souhaitée par C. Benech, nous nous sommes accordés pour que je prenne en charge leur étude. 


\section{ÉTUDE ARCHÉOLOGIQUE : RÉSULTATS DES FOUILLES ET RÉFLEXIONS ISSUES DE L'OBSERVATION DU TERRAIN}

Les diverses interventions survenues sur les fortifications au cours de l'existence de Cyrrhus ont eu pour conséquence de rendre leurs vestiges les plus anciens difficilement visibles. Cependant, avant les travaux de la mission syro-libanaise, la mission française ${ }^{11}$ avait repéré une construction en polygonal à la base des murailles, surmontée d'élévations appartenant à des phases chronologiques postérieures. Cette construction avait été localisée le long de la courtine sud de la citadelle (fig. 2), à l'extérieur, ainsi que sur les remparts méridional et oriental de la ville et, enfin, à la porte septentrionale. Pour E. Frézouls qui l'interprétait comme le vestige d'une première muraille, des parallèles avec Séleucie de Piérie permettaient d'associer cette technique de construction à l'époque hellénistique ${ }^{12}$. Si cette hypothèse paraît très vraisemblable, les précédents fouilleurs n'ont cependant pas pu s'appuyer sur une stratigraphie datée fiable et ni leurs publications, ni leurs rapports ${ }^{13}$ ne délivrent d'information précise sur les résultats des travaux qui ont été entrepris.

\section{Le rempart sud de la citadelle}

Les fouilles que j'ai menées sur la citadelle de 2007 à 2011 ont débuté par l'étude du rempart méridional, choisi en premier lieu car plusieurs tronçons de l'appareil polygonal y étaient apparents. Ils étaient visibles le long de la face externe des courtines, ainsi que dans un ancien sondage d'E. Frézouls situé au pied du rempart entre les tours 2 et 3 (à $20 \mathrm{~m}$ à l'ouest de la tour 3, voir fig. 9). Cet ancien sondage a alors été réétudié mais n'a pas fourni d'informations exploitables ${ }^{14}$. Il a donc été étendu vers l'est (fig. 3), tout en préservant une berme intermédiaire, pour tenter de relier la stratigraphie à la maçonnerie en

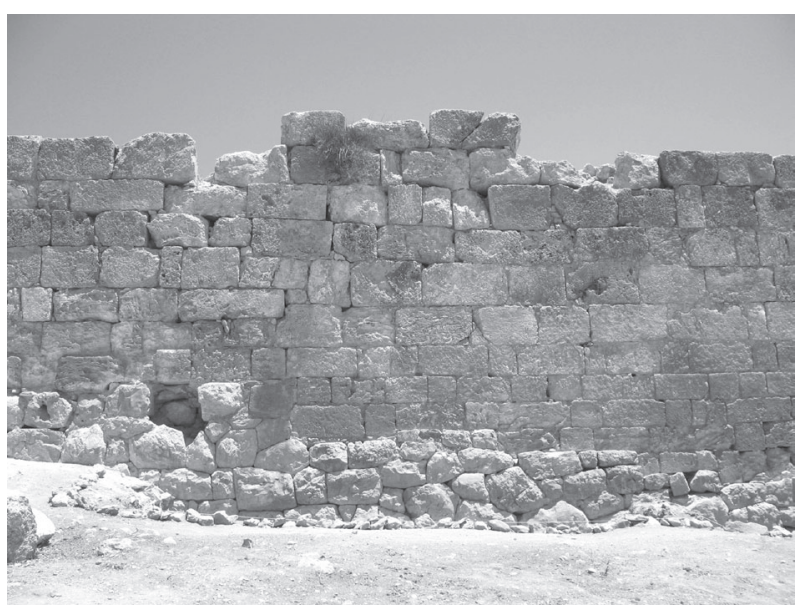

Figure 2. Citadelle, rempart méridional, parement externe (vue vers le nord). L'appareil en polygonal compose le socle avec paliers, parfois altérés ; l'élévation est postérieure (période byzantine) (C) Mission syro-libanaise de Cyrrhus-Nebi Houri.

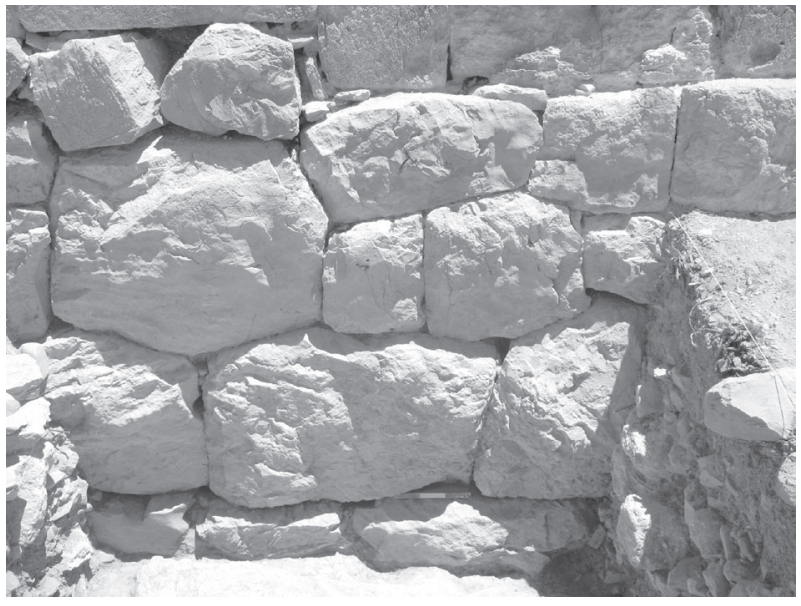

Figure 3. Sondage au pied de la muraille à l'extérieur de la citadelle, détail du parement du socle en polygonal dont le sommet comporte un palier. La maçonnerie repose directement sur le rocher

(C) Mission syro-libanaise de Cyrrhus-Nebi Houri.

11. Le site a été fouillé à partir de 1952 par une mission française dirigée par E. Frézouls.

12. FrÉZOUls 1977, p. 179 et 191.

13. Documents d'archives, non publiés, collection particulière.

14. Au pied du rempart, le sondage avait été poussé jusqu'au rocher et les bermes étaient par trop effondrées pour permettre une exploitation efficace de leur stratigraphie. Les découvertes issues de ce sondage n’ont pas été publiées par la précédente mission, dont les cahiers de fouille ne fournissent pas d'information sur les résultats. 
polygonal. Malheureusement, aucun mobilier archéologique n'y a été retrouvé qui puisse être directement associé à cette maçonnerie, ne permettant pas d'assurer sa date exacte. Il est à noter qu'aucun vestige de construction ni aucun autre élément anthropique antérieur n'a été décelé. En revanche, un niveau de circulation établi le long du mur (fig. 4) représente la première occupation du secteur après la mise en place de la maçonnerie : dans les deux sondages, le niveau repose par endroits directement sur le rocher et à d'autres, sur une couche intermédiaire unique qui comble les irrégularités de la roche naturelle. Or, ce niveau a fourni de la céramique qui remonte à l'époque hellénistique ${ }^{15}$ et était recouvert et en partie détruit par des occupations associées à la période romaine, représentée ici par des sépultures implantées au pied du mur.

Partant du constat que la courtine postérieure repose sur la construction en polygonal, et postulant que la logique des bâtisseurs avait pu être la même pour le choix de la mise en place des tours tardives, j'ai décidé de procéder à la fouille de la tour $3^{16}$, ennoyée sous son propre effondrement. Le mobilier archéologique, découvert en grande quantité dans ses niveaux d'occupation et ses tranchées de fondation, a permis de la dater de la période byzantine. La tour 3 se projette uniquement vers l'extérieur ${ }^{17}$; son élévation

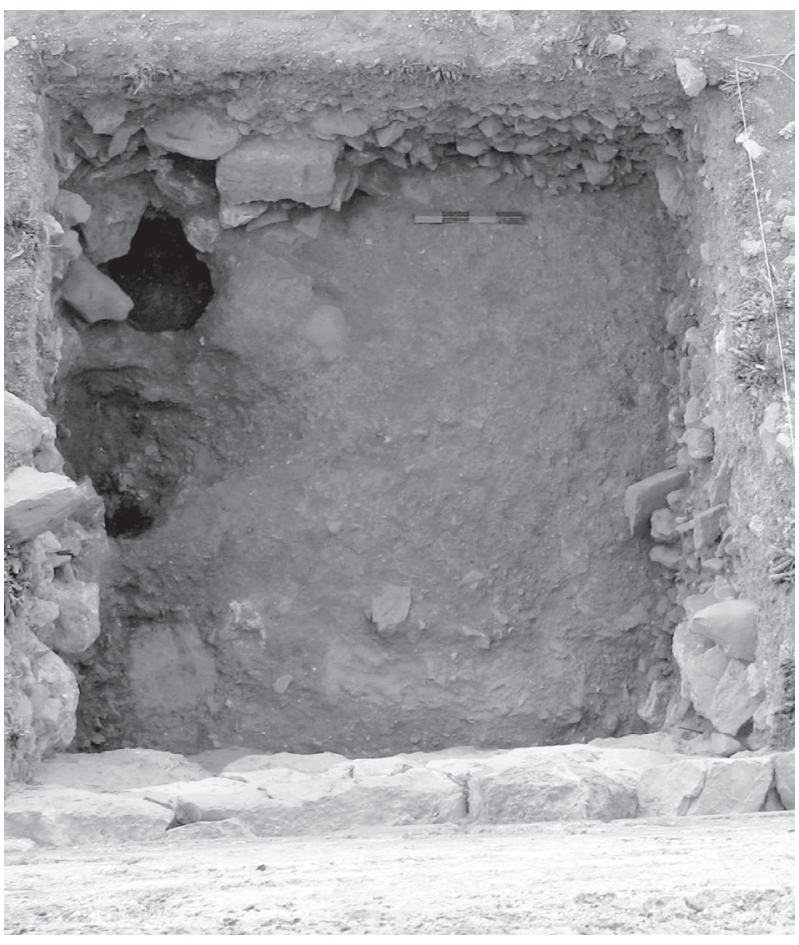

Figure 4. Sondage au pied de la muraille à l'extérieur de la citadelle, vu du haut de la courtine (le nord est en bas); noter l'épaisseur du socle, supérieure à celle de l'élévation, postérieure. Le niveau dégagé, qui pose sur une fine couche de cendres, représente le premier niveau de circulation après la construction de la maçonnerie en polygonal. La zone plus claire au pied du rempart est la roche qui affleure, les fosses à l'ouest sont des vestiges de sépultures romaines

(C) Mission syro-libanaise de Cyrrhus-Nebi Houri. est liée à celle des courtines et bâtie au moyen des mêmes matériaux et techniques, autorisant d'associer également le rempart à la période byzantine. Mais la fouille des abords nord de la tour byzantine et les sondages établis à l'intérieur ont montré qu'elle est fondée sur une tour antérieure (fig. 5 à 7). Cette dernière est de plus grande dimension, et sa base était érigée en appareil polygonal présentant les mêmes caractéristiques que la maçonnerie des courtines étudiées au préalable. Le mur oriental de la tour byzantine s'appuie sur cette première tour (fig. 5) mais en d'autres endroits (murs nord et ouest), la tour byzantine a partiellement détruit la tour en polygonal (fig. 7) dont elle a remployé plusieurs blocs en fondation, liés par un mortier de chaux mêlée de cendres, mortier caractéristique des fortifications de la période byzantine à Cyrrhus.

Cette première tour 3 est implantée à cheval sur le rempart, son mur nord, où se trouve l'entrée, faisant une saillie de 2,50 m vers l'intérieur de la citadelle (fig. 6-7); la partie sud se situe en dehors $\mathrm{du}$ secteur de fouille, mais on peut restituer à cette tour une longueur totale de $16 \mathrm{~m}$ par comparaison

15. Parmi le mobilier découvert dans cette couche, S. Élaigne, qui a pu avoir un aperçu de la céramique, a identifié les tessons comme appartenant à l'époque hellénistique, sans qu'un moment précis de cette période ne se détache plus particulièrement (étude non publiée). Il est à préciser que l'étude du mobilier archéologique était en cours et n'a pu être achevée avant 2011, date à partir de laquelle la mission n'a plus pu avoir accès au matériel.

16. À partir de 2007, fouille achevée en 2010.

17. Ses dimensions externes sont de 8,60 m E-O x 8,30 m N-S, pour des dimensions internes de 3,45 m E-O x 5,90 m N-S. 


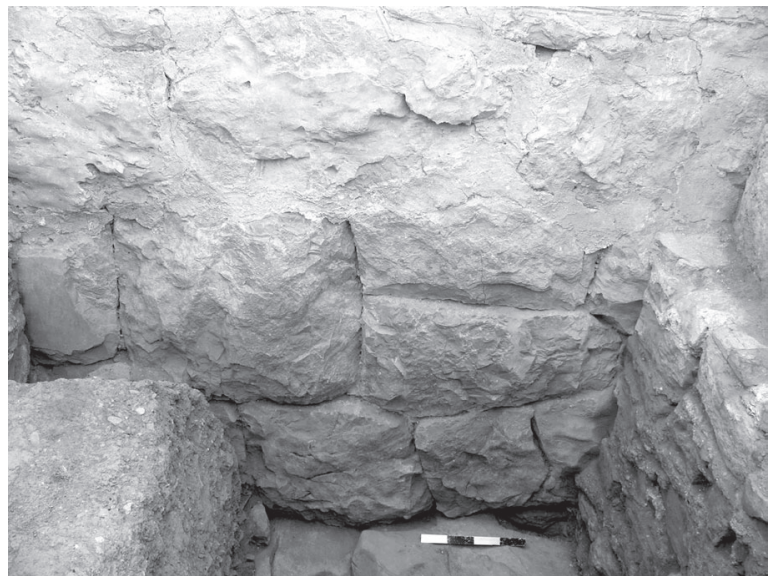

Figure 5. Assises de la première tour 3 en polygonal, sous l'élévation de la tour byzantine (vue vers l'est). La mire repose sur le rocher (c) Mission syro-libanaise de Cyrrhus-Nebi Houri.

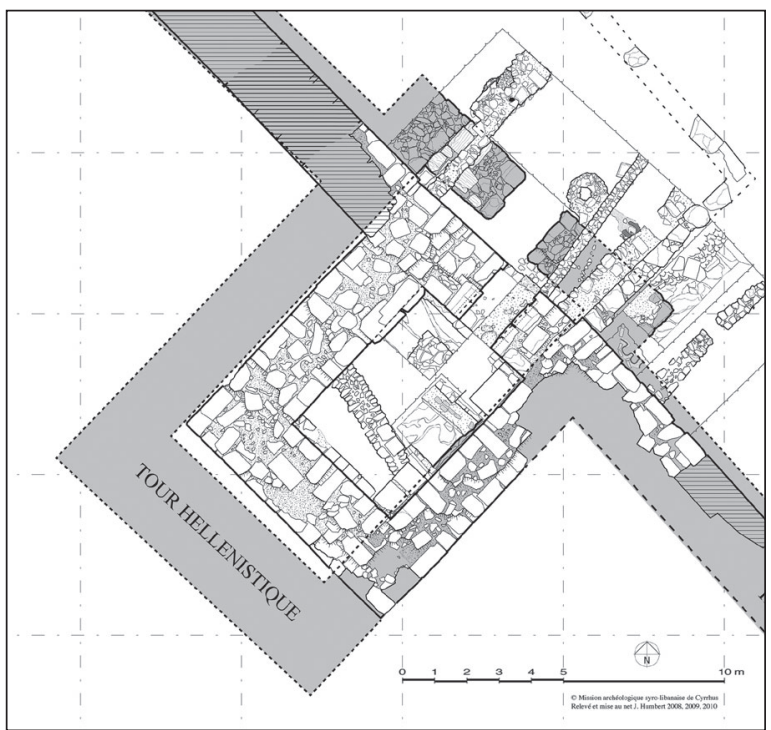

Figure 6. Plan de la tour en polygonal (en gris) sous la tour postérieure. En tiretés, le tracé supposé ; en traits pleins, le tracé retrouvé. Le mur oriental de la tour byzantine repose sur celui de la tour antérieure.

Relevé et mise au net J. Humbert

(C) Mission syro-libanaise de Cyrrhus-Nebi Houri.

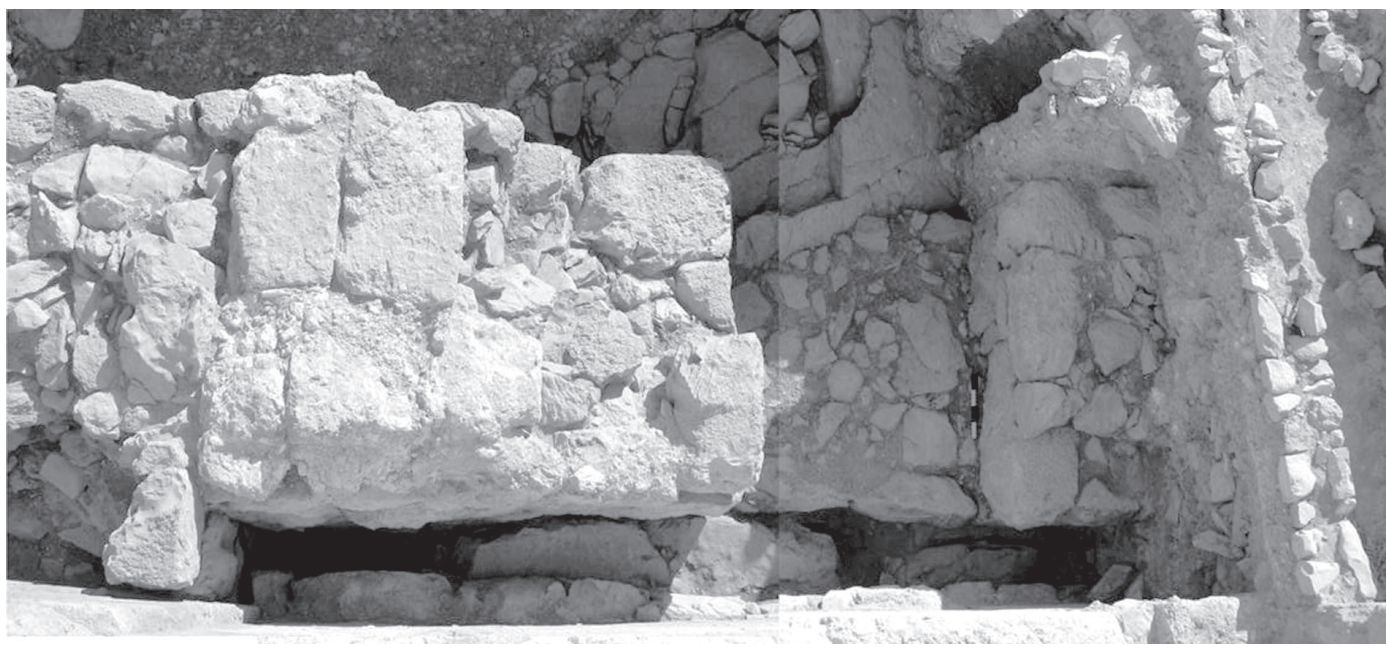

Figure 7. Le mur nord et la porte de la première tour 3 en polygonal, vus du sommet de la tour byzantine (dont le mur nord apparait en bordure basse du cliché). Le nord est en haut. La mire est posée dans le passage, sur le sol de fonctionnement empierré. La partie orientale de la porte s'enfonce sous la berme, à droite du cliché ; la partie occidentale (à gauche sur le cliché) a été détruite pour les besoins de la mise en place de la tour byzantine, mais on voit son angle interne nord-ouest se dessiner. En son centre, cette partie occidentale conserve également des vestiges de l'assise de réglage de l'élévation, en blocs de calcaire tendre, immédiatement à l'est de l'angle interne de la tour. (c) Mission syro-libanaise de Cyrrhus-Nebi Houri. 
avec celle de la tour $37^{18}$, de mêmes factures, caractéristiques et dimensions. Son mur est a tranché des niveaux de terre naturelle, dans la pente vers le sud, qui ne présentaient aucune trace d'occupation antérieure. Le niveau de circulation de la porte de la première tour 3 (fig. 7) a connu un abandon de courte durée ${ }^{19}$, puis une réoccupation associée à un épais niveau de fonctionnement ${ }^{20}$ situé au nord de la tour, qui a pu être datée du début de l'époque romaine, au $\mathrm{I}^{\mathrm{er}} \mathrm{s}$. av. J.-C.

Ces travaux sur la courtine et sur la tour m'ont permis de constater que cette construction en appareil polygonal représente le socle ${ }^{21}$ de fortifications dont l'élévation d'origine a disparu, remplacée par celle d'époque byzantine (fig. 2), plus étroite que le socle initial (fig. 4) ${ }^{22}$. Lorsque la partie basse du socle s'est retrouvée, au fil des siècles et en certains secteurs, partiellement enterrée, cette maçonnerie a pu devenir la fondation des élévations d'époques postérieures. À l'intérieur de la première tour 3, sur sa face interne orientale, la maçonnerie en polygonal représentait également sa fondation, dans la mesure où elle était en partie enterrée ; mais elle demeurait entièrement visible à l'extérieur, différence due au dénivelé de la pente sur laquelle est implantée la tour.

Le socle est une maçonnerie massive, épaisse de $2,70 \mathrm{~m}$ pour les courtines et de $2 \mathrm{~m}$ pour la tour, haute de 2 à $3,30 \mathrm{~m}$ pour les courtines contre une hauteur de 1 à $1,70 \mathrm{~m}$ à la base de la tour. Elle est faite de blocs polygonaux de plusieurs tailles ${ }^{23}$ disposés en parement, à joints secs, qui maintiennent un remplissage de tout-venant lié par une terre rouge argileuse compacte (fig. 6-7). La pierre est le calcaire dur local, extrait en surface à proximité immédiate des fortifications ${ }^{24}$; les blocs ont été retouchés de façon à être parfaitement jointifs (les zones de contact laissent entrevoir des piquetages), ainsi que ceux situés au sommet du socle des courtines et de la tour, qui présentent une surface plane. Leur mise en place s'est opérée directement sur le rocher et parfois, celui-ci a été creusé de légères entailles (quelques centimètres de profondeur), visibles sur un seul côté de la construction du fait du dénivelé naturel, de manière à former des plateformes horizontales pour asseoir le socle.

Le sommet du socle des courtines forme des paliers horizontaux disposés « en escaliers » de façon à s'adapter à la pente, ce qui a contraint les constructeurs byzantins à employer des blocs taillés de diverses dimensions afin d'ajuster l'élévation aux dénivelés causés par ces paliers (fig. 2 et 3 ). Un palier a également été repéré sur le mur nord-ouest de la tour.

Le fait que le sommet du socle soit plan montre bien que l'appareil en polygonal ne se poursuivait pas en hauteur, mais que des blocs régulièrement taillés prenaient place au-dessus. L'élévation d'origine, encore visible sur le mur nord de la première tour 3, était en blocs de calcaire tendre liés au mortier blanc ${ }^{25}$ (fig. 7). Dans ce cas précis, il s'agit de vestiges de l'assise de réglage, de tailles diverses, afin d'adapter l'élévation au relief formé par l'appareil polygonal à l'emplacement du palier de compensation. Cependant, dans l'élévation de la muraille postérieure, plusieurs blocs en calcaire tendre sont remployés, contrastant avec la majorité des pierres utilisées à Cyrrhus aux époques romaine et byzantine, lesquelles entrent dans la catégorie des pierres froides, soit d'une grande dureté ${ }^{26}$. Ces blocs de pierre tendre présentent également la caractéristique d'être de dimensions modulaires correspondant à des tailles

18. Voir plus bas.

19. Pierres tombées dans le passage de la tour, puis niveau égalisé. La durée exacte de cet abandon n'est pas connue.

20. Couche de $50 \mathrm{~cm}$, immédiatement au nord de l'édifice, à l'intérieur de la citadelle.

21. Je préfère le terme de "socle » à celui de «soubassement», considérant son emploi comme « socle de mur» et non « socle de bâtiment» mais aussi qu'il peut comprendre une différence de matériaux avec l'élévation et n'implique pas la présence d'une fondation. Enfin, ce terme rend également mieux compte de la maçonnerie dans sa massivité, quand « soubassement» peut ne concerner que ses seuls parements. Je remercie J.-C. Bessac pour les échanges que nous avons pu avoir sur ce sujet.

22. Jusqu'à $30 \mathrm{~cm}$.

23. Les plus grands ont une longueur jusqu'à $1,20 \mathrm{~m}$ et / ou une hauteur de $60 \mathrm{~cm}$.

24. Observations menées et aimablement transmises par J.-C. Bessac sur le terrain. Voir également BESSAC 2016, p. 132.

25. Je n'ai pas, sur place, déterminé la nature du mortier, plâtre ou chaux. La roche présente à Cyrrhus et dans la région laisse cependant envisager que le deuxième matériau est le plus probable.

26. Information aimablement transmise par J.-C. Bessac sur le terrain. Voir également BESSAC 2016, p. 132. 


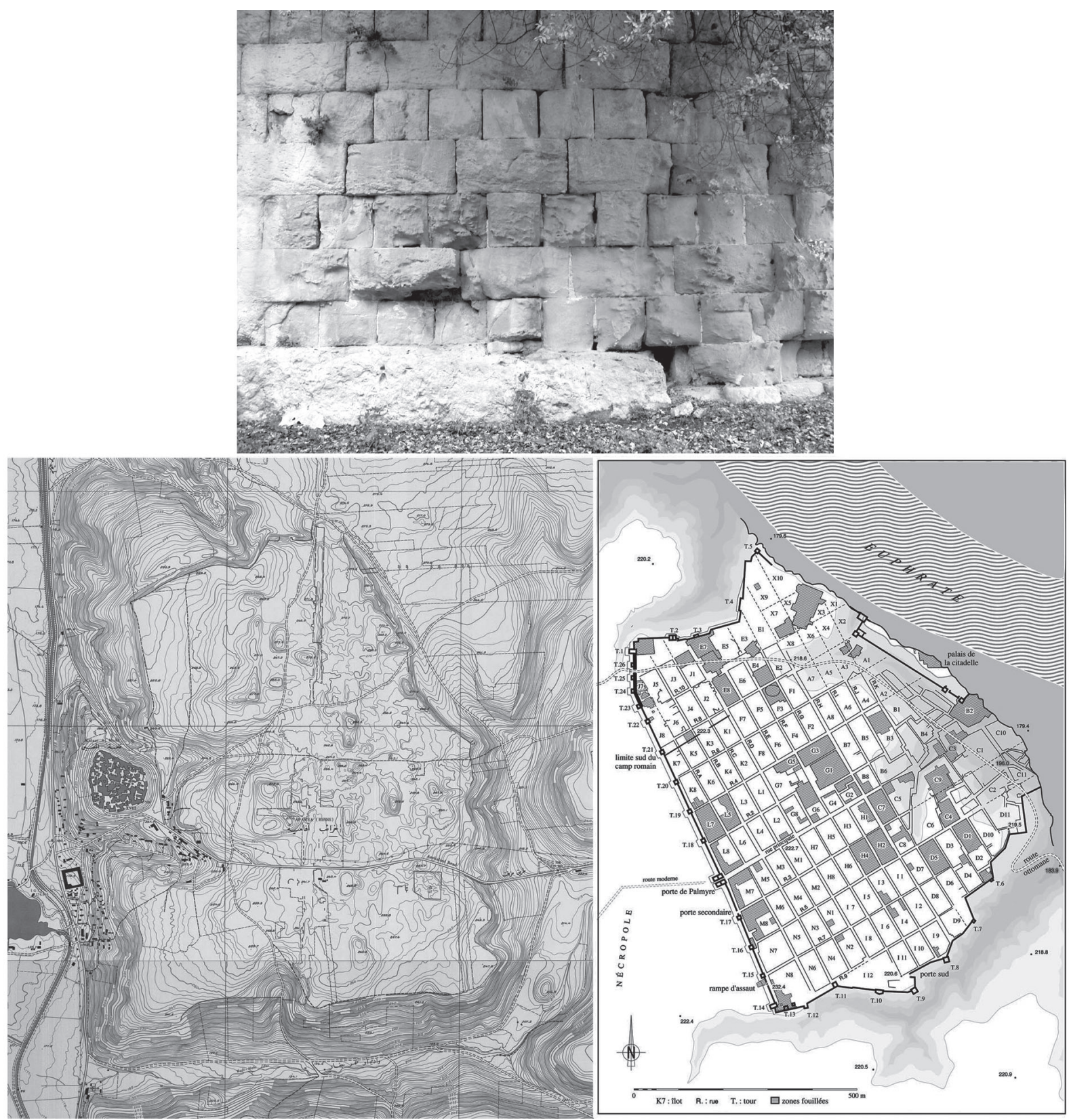

Figure 8. Exemples comparatifs :

En haut, Séleucie de Piérie, élévation du rempart

en blocs modulaires de pierre tendre (C) M. Gelin.

En bas à gauche, plan d'Apamée de l'Oronte et de sa citadelle Qalaat al Mudiq :

la ville est implantée sur un plateau bordé de falaises, les fortifications ont été établies

en limite du plateau (exploitation du relief à des fins défensives) ; carte syro-italienne (carroyage : 100m)

(C) ministère syrien des Travaux publics, 1963.

En bas à droite, plan de Doura-Europos sur l'Euphrate : sur le rempart occidental,

les tours d'angles 1 , au nord, et 14, au sud, sont de grandes dimensions pour couvrir des angles de vue et de tirs importants, en comparaison avec les tours alignées sur ce rempart, qui sont de dimensions moindres ; noter également les tracés des murailles nord, sud et est, qui s'alignent sur les bordures de falaises ; plan H. David-Cuny

(C) Mission archéologique franco-syrienne de Doura-Europos. 
courantes à la période hellénistique ${ }^{27}$. Ces deux particularités m'ont permis de les associer à une tradition de fortifications hellénistiques en Syrie, comme on le voit à Ibn Hani, Qalaat al Mudiq et Apamée de l'Oronte, Séleucie de Piérie (fig. 8) ou encore à Doura-Europos sur l'Euphrate ${ }^{28}$, et de supposer qu'ils proviennent de la première élévation, tant celle de la tour que des courtines. Cette hypothèse, élaborée au vu des éléments présents à la citadelle, a rapidement été confirmée lors de la fouille de la tour 37 (voir plus bas).

Si l'on connaît désormais la nature de la construction en polygonal, en revanche, tout comme dans le cas de nos prédécesseurs, aucun mobilier archéologique n'a été retrouvé permettant de dater les socles de manière absolue. Néanmoins, la céramique découverte dans les niveaux d'occupation immédiatement postérieurs à la première tour, permet de les situer avant le $\mathrm{I}^{\mathrm{er}}$ siècle av. J.-C., soit avant la présence romaine à Cyrrhus, et les tessons de céramique trouvés dans le premier niveau d'occupation postérieur au socle de la courtine offrent un terminus ante quem de l'époque hellénistique. L'emploi des blocs modulaires en pierre tendre permet également d'y associer la construction.

C'est avec cette hypothèse de travail qui lie le rempart en polygonal à l'époque hellénistique que j'ai poursuivi mes recherches. Je me suis attachée tout d'abord à l'étude de vestiges d'une maçonnerie préservés à l'est de la citadelle, sur la muraille méridionale descendant vers la ville basse.

\section{Le rempart sud-ouest de la ville hellénistique}

La mission syro-libanaise a pu constater la présence des vestiges de la muraille hellénistique

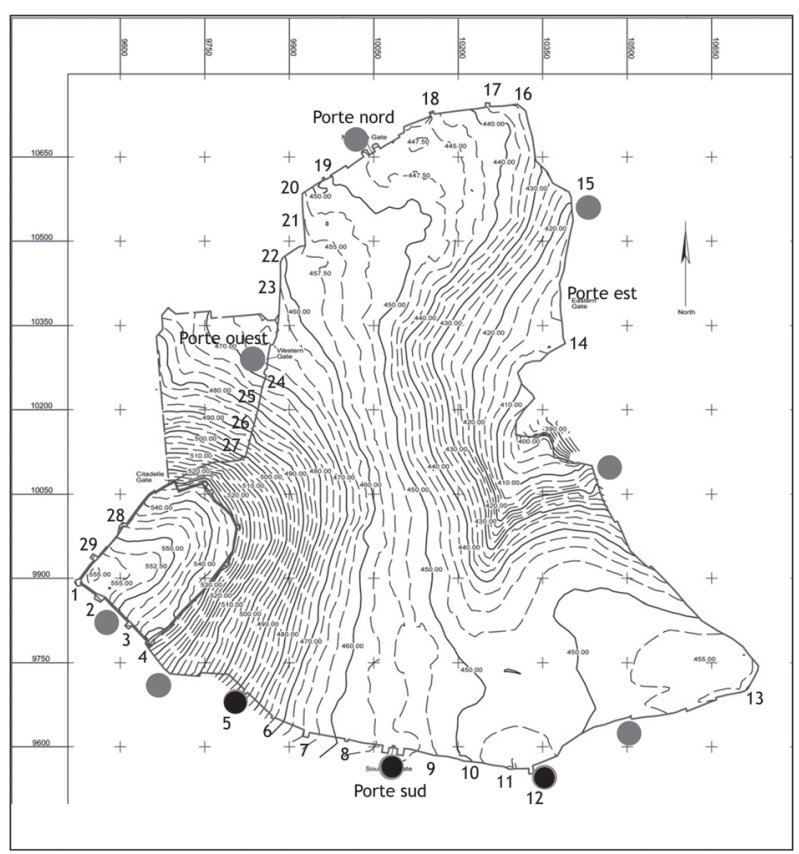

Figure 9. Plan de Cyrrhus, avec indication des secteurs du rempart où le socle en polygonal a été repéré (en gris) et où il est absent (en noir). Le carroyage est de $150 \mathrm{~m}$.

Relevé et mise au net S. Baier et S. Knechtel (C) Mission syro-libanaise de Cyrrhus-Nebi Houri.

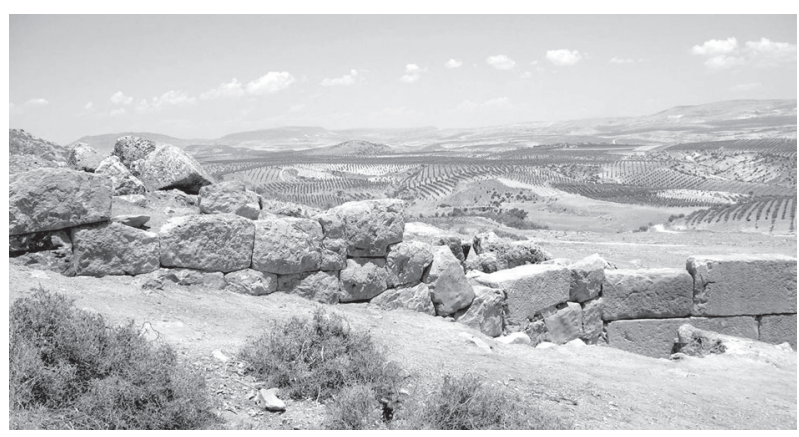

Figure 10. Jonction du rempart hellénistique en polygonal et du rempart byzantin en blocs rectangulaires de remploi, immédiatement à l'ouest de la tour 5 (vue vers le nord-est)

(C) Mission syro-libanaise de Cyrrhus-Nebi Houri. sur l'extrémité orientale du rempart sud de la ville ${ }^{29}$. En revanche, à la porte sud ${ }^{30}$ elle n'a pu que constater son absence, tout comme sur la majeure partie du tracé méridional depuis, d'ouest en est, un coude opéré par la muraille à environ $40 \mathrm{~m}$ à l'ouest de la tour 5 jusqu'à un deuxième changement de direction de la muraille à l'est de la tour 12 (fig. 9). De fait, l'actuelle muraille sud de la ville, en dehors de ses extrémités occidentale et orientale, est fondée sur un tracé d'époque romaine, repris aux époques byzantine et islamique ${ }^{31}$.

27. Dimensions approchant $102 \times 54 \mathrm{~cm}$, ce qui peut être interprété comme 2 x 1 coudées pour une hauteur d'assise de 47-48 cm (épaisseur probablement dictée par celle des bancs rocheux d'où la pierre a été extraite).

28. Pour Doura-Europos, Qalaat al Mudiq, Apamée de l'Oronte et Ibn Hani, voir Bessac 1997, Gelin 2007, et Leriche 2000.

29. Recherches de Sh. Al-Shbib, publication à venir.

30. Recherche de J. Abdul Massih, publication à venir.

31. Abdul Massin 2012. 
Partant de ce constat, il fallait déterminer où se trouvait le tracé du premier rempart méridional.

Immédiatement à l'est de la citadelle, entre la tour 4 et le changement de direction proche de la tour 5, se trouve un alignement de blocs polygonaux qui descend vers la ville, formant un redan en pointe qui se projette vers l'extérieur puis poursuit son tracé vers l'est (fig. 9). Le rempart forme ensuite un décrochement, réorientant son tracé vers l'intérieur de la ville, vers le nord-est. Puis, à environ $150 \mathrm{~m}$ à l'est du mur oriental de la citadelle, le mur en appareil polygonal disparaît, laissant la place à un appareil en blocs rectangulaires de diverses dimensions, probablement des remplois (fig. 10). La jonction entre les deux murs se fait dans un rentrant du tracé et le rempart en blocs rectangulaires se dirige vers le sud-est, vers l'actuelle porte méridionale de la ville.

À l'endroit où disparaît le rempart en polygonal et, suivant la logique de l'implantation de son tracé qui s'oriente vers le nord-est, j'ai recherché dans cette direction l'éventuelle présence de blocs polygonaux. Effectivement, plusieurs apparaissaient en surface (fig. 11), en partie masqués par la végétation. Ces blocs voisinent avec le rocher qui affleure en différents endroits, la pente ayant été fortement lessivée par les intempéries. Le nettoyage des blocs et le désherbage des niveaux de surface sur le prolongement de leur tracé ${ }^{32}$ ont confirmé la présence de deux alignements parallèles descendant vers la ville basse, vestiges des parements de la muraille. Cependant, la jonction de ce tracé avec celui de la maçonnerie en polygonal en place plus à l'ouest, dans les parties hautes de la pente, ne s'opérait pas selon une ligne droite mais plutôt, si on les avait prolongées, selon deux lignes parallèles. C'est pourquoi j'ai choisi d'établir un sondage de part et d'autre de la jonction entre le rempart en blocs polygonaux et le rempart en blocs rectangulaires (fig. 12). À l'intérieur de la ville, le rocher est immédiatement apparu, portant parfois des traces de piquetage : ces zones, qui ont aplani le terrain pour former autant de petites plateformes horizontales, correspondent à des entailles de fondation destinées à des blocs disparus, alignées avec quelques blocs toujours en place, qui composaient les parements du socle de la muraille. Il a ainsi été possible de restituer l'ensemble du tracé du premier rempart, et de constater qu'il formait là aussi un décrochement identique à celui repéré plus haut dans la pente (fig. 12-13).

En suivant la muraille en contrebas, à environ $50 \mathrm{~m}$ au nord-est de ce décrochement, la mission a mis au jour une imposante tour $37^{33}$ (environ 12 × 16 m, fig. 14). Elle est bâtie en appareil polygonal semblable à celui déjà dégagé à la citadelle et dans la pente, et se trouve à cheval sur le rempart; sur sa courtine orientale, l'élévation en blocs de calcaire tendre modulaires était encore conservée sur plusieurs assises. Sa construction et ses dimensions, dont la largeur est identique à celle de la tour 3 , nous ont permis de restituer le plan de cette dernière. Enfin, à également une cinquantaine de mètres au sud-est de la nouvelle tour 37, divers alignements de blocs polygonaux ont permis à J. Humbert de restituer une tour 38, de mêmes dimensions que les deux précédentes (fig. 15).

De plus, l'actuelle tour d'angle sud-est de la citadelle (tour 4) et les deux décrochements qui lui succèdent dans la pente sont régulièrement espacés, d'une distance d'environ $50 \mathrm{~m}$, que J. Humbert restitue de 100 coudées. Sur le plan des vestiges qu'il a réalisé, il a montré de manière convaincante que sous la tour 4 pouvait prendre place également un décrochement, ce qui permet de relier le tracé de la courtine menant à la première tour 3 (fig. 15). On peut ainsi restituer, de part et d'autre du saillant, un tracé des fortifications en dents de scie.

Le premier rempart méridional hellénistique prend donc la forme de courtines séparées tous les $50 \mathrm{~m}$ environ par des décrochements ${ }^{34}$ ou par de grandes tours. En partie sur la citadelle, dans son étendue actuelle, et en partie sur la pente menant vers la ville basse, trois tours ont été dénombrées ainsi que trois

32. Le secteur a été dégagé par une partie des chefs de chantiers de la mission, J. Abdul Massih, A. Kannawi et Sh. Al Shbib (et l'ensemble de leurs ouvriers), qui ont ponctuellement délaissé leurs propres travaux afin de me permettre d'obtenir plus rapidement les résultats, ce dont je les remercie.

33. La tour a été fouillée par J. Abdul Massih, A. Kannawi et Sh. Al Shbib.

34. Tracé en crémaillère. Voir GINOUvÈs 1998, p. 26. 


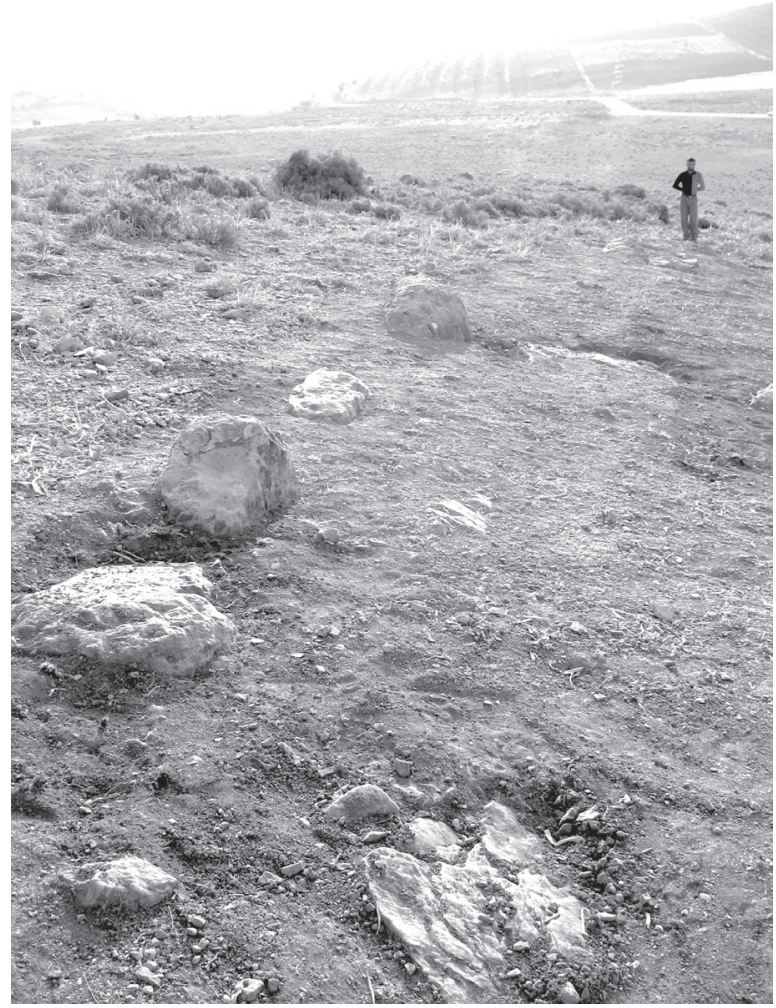

Figure 11. Les blocs polygonaux retrouvés dans la pente (ici désherbée), au nord-est du " changement de direction » (vue vers le nord-est). Au premier plan, affleurement du rocher (C) Mission syro-libanaise de Cyrrhus-Nebi Houri.

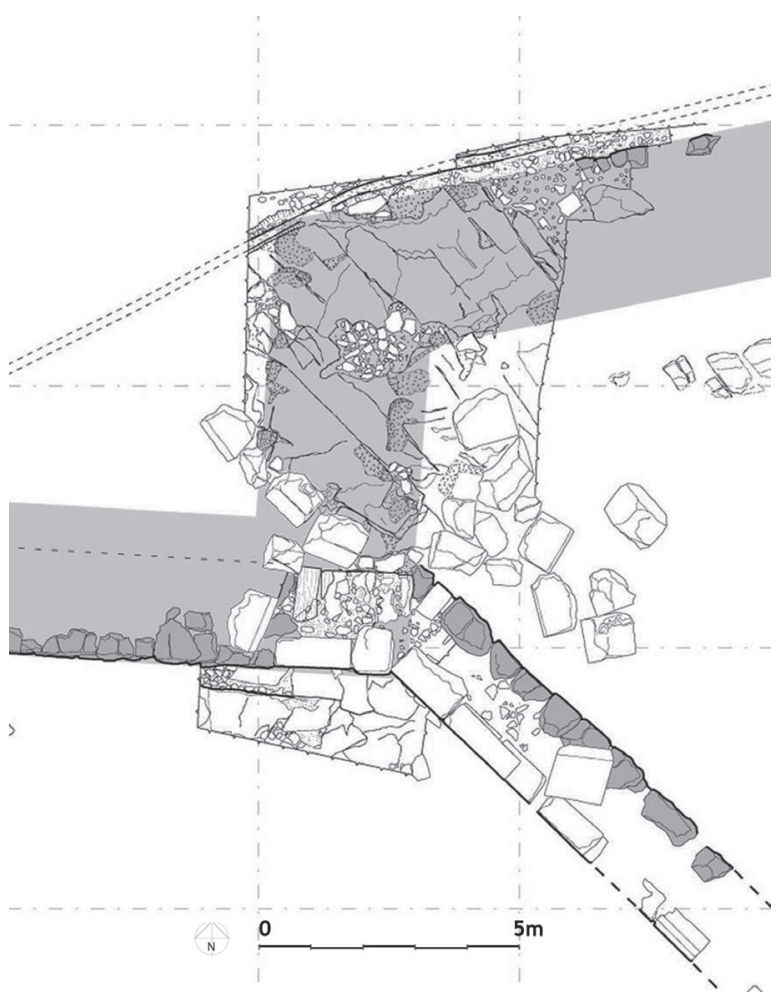

Figure 12. Dans la pente de la citadelle vers la ville, sondage implanté sur le changement de direction du rempart, qui a permis de restituer un décrochement du mur d'origine. En gris clair, le tracé restitué du rempart d'origine ; en gris foncé, les blocs polygonaux, en place sur le rempart d'origine (à gauche), ou remployés en parement sur le rempart byzantin (en bas à droite). En pointillés, les traces de piquetage du rocher pour l'implantation du mur en polygonal. Relevé et mise au net J. Humbert (C) Mission syro-libanaise de Cyrrhus-Nebi Houri.

Figure 13. Vue aérienne vers l'ouest montrant le deuxième décrochement du rempart en appareil polygonal (à gauche au centre du cliché) et, en bas, le sondage implanté à la jonction de la muraille hellénistique venant de la citadelle avec la muraille byzantine descendant vers la ville basse. Le troisième décrochement du tracé de l'enceinte en polygonal y est apparu. On voit dans la pente à droite quelques blocs polygonaux, vestiges des parements de la muraille. En haut, le mur oriental de la citadelle, à gauche, la tour 4 . Photo Y. Guichard (C) Mission syro-libanaise de Cyrrhus-Nebi Houri.

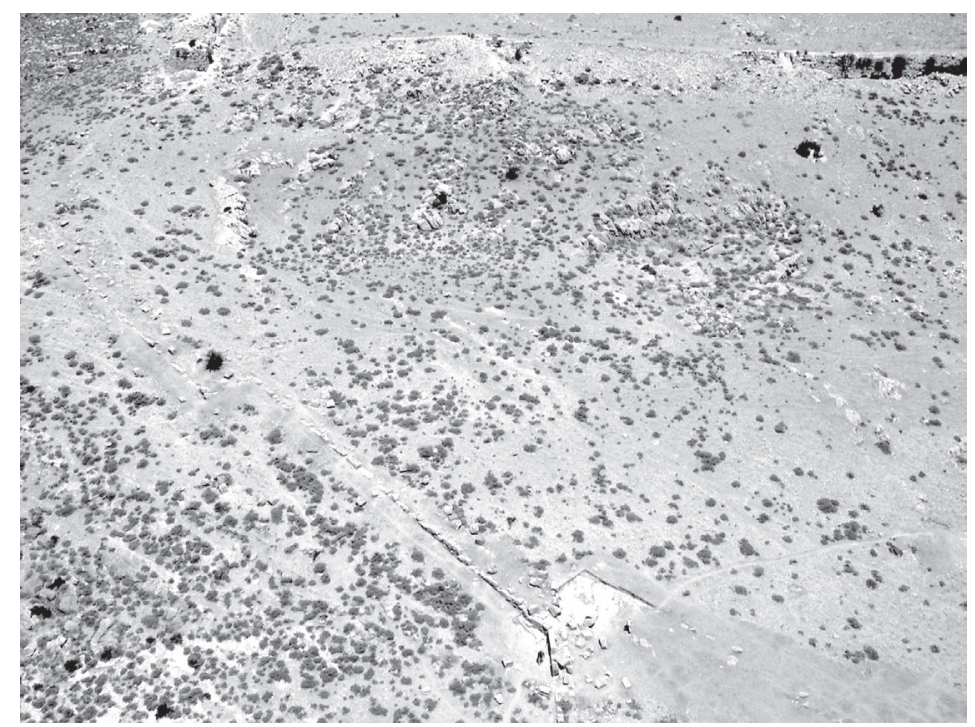




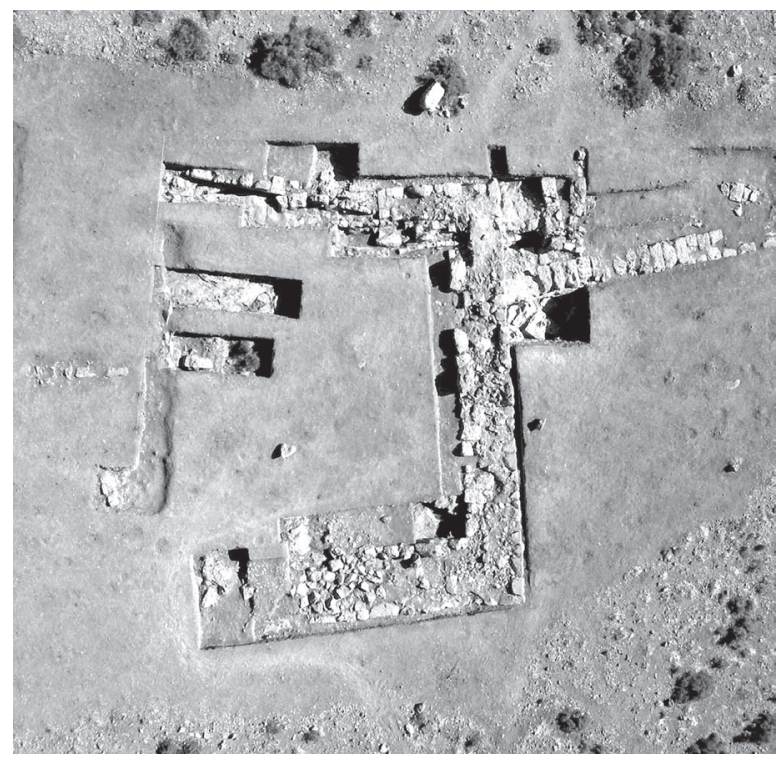

Figure 14. La tour 37 dégagée dans la pente, dans le prolongement du rempart en polygonal vers le nord-est (le nord est en haut). Sur la courtine partant vers le nord-est, blocs modulaires qui composaient l'élévation. Photo Y. Guichard

(C) Mission syro-libanaise de Cyrrhus-Nebi Houri.

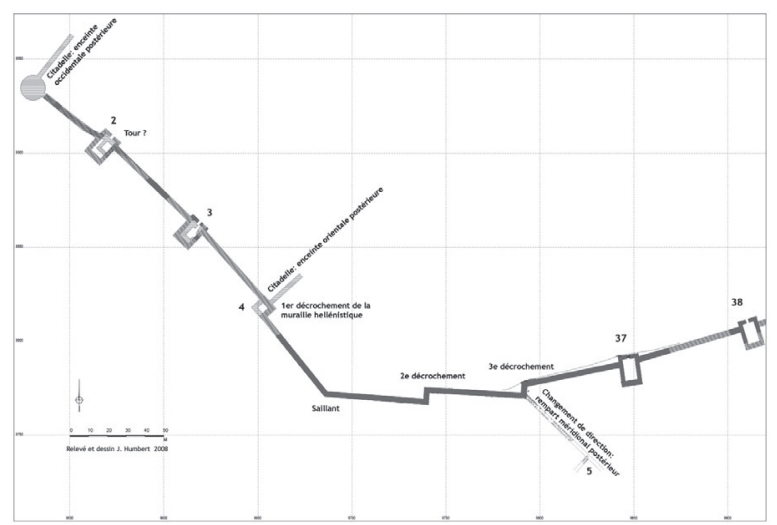

Figure 15. En gris foncé, proposition de restitution du tracé du rempart en appareil polygonal sur la partie occidentale des fortifications méridionales (citadelle et pente). En gris clair, les murailles postérieures. Le carroyage est de $50 \mathrm{~m}$. Plan J. Humbert

(C) Mission syro-libanaise de Cyrrhus-Nebi Houri.

décrochements et un saillant. Il est envisageable de restituer une tour sous l'actuelle tour 2 ainsi que sous la tour d'angle sud-ouest de la citadelle (tour 1) ${ }^{35}$.

Il est désormais clair que le rempart en appareil polygonal a été implanté en respectant les lignes du relief : dans les zones les plus élevées, il se situe en rupture de pente et, lorsque ce relief forme une courbe vers le nord-est, la muraille prend la même orientation. Dans la ville basse, au point le plus bas au centre de la courbe « rentrante » formée par les lignes du relief, J. Abdul Massih a implanté un sondage qui a révélé un seuil marquant une porte, qui pourrait être liée à ce rempart ${ }^{36}$. À partir de ce point, si l'on s'en tient à la même logique suivie par les constructeurs de la muraille en polygonal et à l'observation du terrain, le rempart devait probablement poursuivre son tracé parallèlement à celui du relief et, tout comme lui, se retourner également vers le sud-est : ceci explique à la fois son absence au centre de l'actuelle muraille méridionale et sa présence dans l'angle sud-est de la ville, au sommet d'un dénivelé dominant la rivière Saboun Souyou. Ces résultats et hypothèses permettent de proposer une première restitution du tracé des fortifications méridionales hellénistiques (fig. 16).

L'ensemble des caractéristiques de ces fortifications, comme leur conception générale ainsi que leur implantation et leurs techniques de construction, se rattache en Syrie antique à une tradition hellénistique systématique de défense urbaine. Ainsi, implanter la muraille sur une rupture de pente permet d'exploiter le relief à des fins de renforcement de la défense. Ce type d'implantation, dans la région et pour la période qui nous intéressent, a été reconnu plus particulièrement sur les fortifications

35. Je n'ai pas eu le temps de procéder à des fouilles de ces secteurs avant 2011. Cette interruption du travail m'a empêchée de restituer l'étendue de la citadelle hellénistique qui n'avait probablement pas les mêmes dimensions que celle que l'on voit aujourd'hui : le tracé du mur oriental de l'actuelle citadelle ne reprend pas celui de courtines antérieures, et son tracé occidental repose sur des blocs polygonaux remployés.

36. Voir la contribution de J. Abdul Massih dans ce volume. 


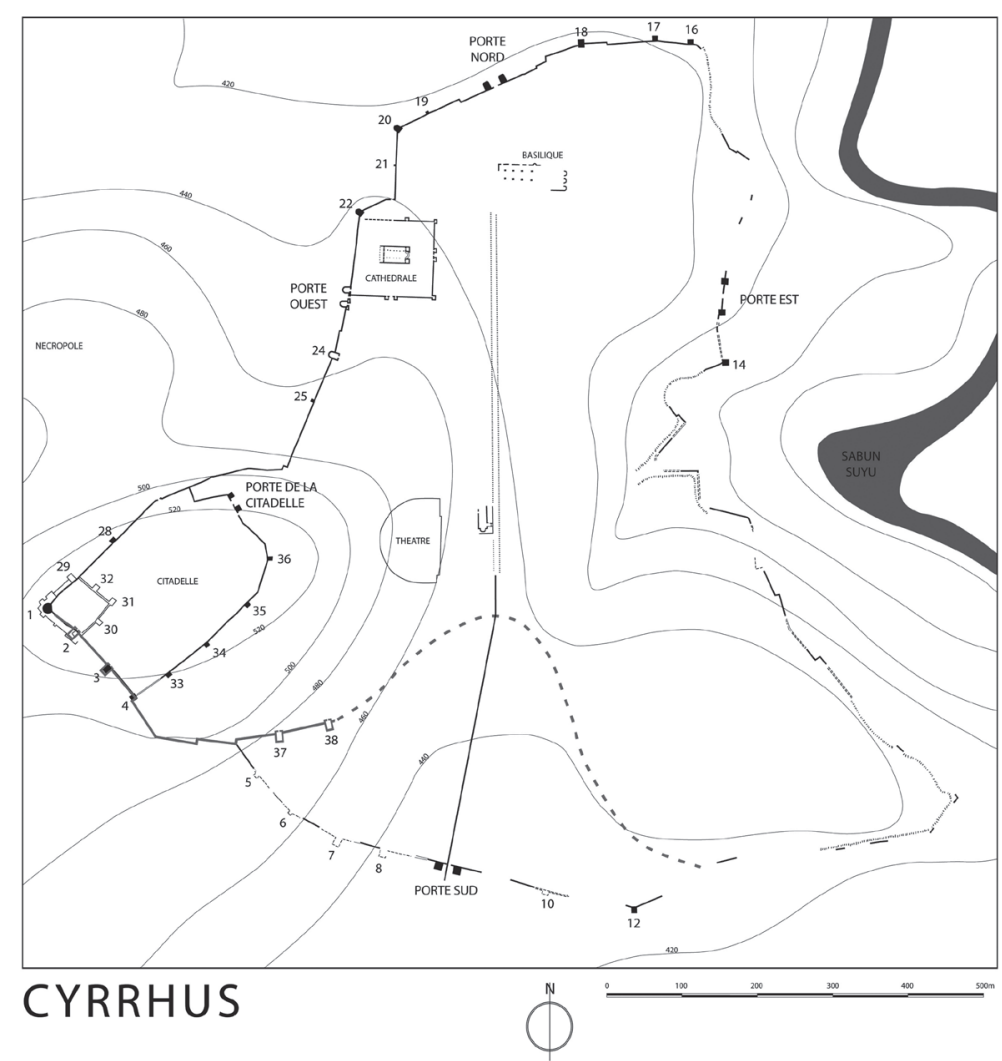

Figure 16. En gris et tiretés, proposition de restitution du tracé de la muraille méridionale d'origine, d'après les résultats des recherches archéologiques (rempart en polygonal et première porte sud).

Dessin M. Gelin sur un plan D. Longepierre.

d'époque hellénistique (Jebel Khalid ${ }^{37}$, Doura-Europos sur l'Euphrate, Apamée de l'Oronte ; fig. 8). L'appareil du socle, de type polygonal, a été employé de manière régulière à l'époque hellénistique : il est similaire notamment à celui de Séleucie de Piérie ou d'Apamée de 1'Euphrate ${ }^{38}$. L'appareil de l'élévation, fait de blocs modulaires de calcaire tendre est, on l'a vu, propre à certaines fortifications hellénistiques de Syrie.

Cependant, ces caractéristiques offrent une fourchette chronologique large qui couvre l'ensemble de la période hellénistique, sans plus de précision. Il en va de même des éléments de datation relative, qui confortent cette association de la muraille en polygonal de Cyrrhus à la période hellénistique, mais sans apporter de date plus claire :

- La muraille, sur la courtine 2-3, peut être associée à l'époque hellénistique (antérieure ou contemporaine) et la tour 3 d'origine est antérieure à la première occupation romaine $\mathrm{du} \mathrm{I}^{\mathrm{er}} \mathrm{s}$. av. J.-C.

- Cyrrhus n'apparaît dans les témoignages antiques qu'à partir de l'époque hellénistique (deuxième moitié du $\mathrm{III}^{\mathrm{e}} \mathrm{S}$. av. J.-C.) et, sur le terrain, aucun mobilier antérieur à cette époque n'a été mis en évidence.

37. Pour Jebel Khalid, voir le plan p. 132 dans Clarke et al. 2011. Pour Doura-Europos, voir également Von Gerkan 1939.

38. Séleucie de Piérie, voir van Berchem 1954, pl. XXIV-2 ; Apamée de l'Euphrate, voir Desreumaux, Gaborit \& Caillou 1999 , fig. 5 et 6, p. 82, et GABORIT 2012, fig. 3, p. 201. 
- L'absence de mobilier archéologique ou autre vestige anthropique préexistant, sur le secteur étudié ${ }^{39}$ mais également partout où les missions française et syro-libanaise ont travaillé à Cyrrhus, et le fait que les murailles en appareil polygonal soient fondées directement sur le rocher sans aucune présence de mobilier associé, permettent de supposer que ces fortifications représentent la première occupation attestée.

Ce faisceau d'indices concordants plaide en faveur du fait que les fortifications en appareil polygonal représentent très vraisemblablement le premier système défensif mis en place à Cyrrhus et qu'il remonte à l'époque hellénistique.

Ainsi, grâce à l'observation du terrain et aux recherches archéologiques, la mission a pu restituer ${ }^{40}$ la majeure partie du tracé du rempart de la ville hellénistique d'origine (fig. 16). Cette première ville occupait une surface certes un peu réduite par rapport à celle de la ville postérieure, mais exploitait totalement les possibilités offertes à sa défense - à la fois active et passive - par les éléments naturels, parmi lesquels le relief a joué un rôle déterminant. L'extension vers le sud, consacrée par le dernier rempart et par l'actuelle porte sud, appartient aux époques romaine et byzantine, avec des réaménagements d'époque islamique ${ }^{41}$.

\section{ÉTUDE DE LA CARTE GÉOPHYSIQUE : ESSAI D’INTERPRÉTATION}

La prospection géophysique a été menée par C. Benech ${ }^{42}$ dans la ville basse, au centre du secteur méridional, chevauchant en partie la ligne supposée des fortifications de la première ville connue. La carte obtenue montre très nettement la continuation du tracé du premier rempart repéré à la citadelle et dans la pente, qui respecte bien le relief et permet de confirmer les hypothèses émises précédemment (fig. 17).

\section{Les courtines et les tours : description}

Sur la muraille occidentale ${ }^{43}$, la courtine située entre la tour 39 et la première porte ${ }^{44}$ mesure une centaine de mètres, mais il est très probable qu'une tour 40 , non visible sur l'image géophysique, y a pris place ${ }^{45}$. Dans cette hypothèse, on pourrait attribuer une longueur d'une cinquantaine de mètres aux courtines 39-40 et 40-première porte.

Là où elles sont le plus visible, c'est-à-dire sur le rempart oriental, les courtines de la ville basse sont légèrement moins longues que celles de la citadelle et de la pente et, surtout, espacées moins régulièrement : la courtine entre la première porte et la tour 41 est de $43 \mathrm{~m}$; la courtine $41-42$ mesure $39 \mathrm{~m}$ et la courtine $42-43,46 \mathrm{~m}^{46}$.

39. Quelques tessons de céramique, qui pourraient remonter à l'âge du Bronze, ont été découverts dans des fragments de briques crues remployés dans le remplissage de la première tour 3, mais aucune installation relevant de cette période n'a été découverte.

40. Proposition émise dès 2008 par l'auteure de ces lignes et J. Abdul Massih.

41. Voir les publications à venir de la mission et AL-SHBiB 2015 et 2017 ; pour le développement de l'urbanisme de cette partie sud de la ville, voir BENECH à paraître.

42. Je remercie tout particulièrement $\mathrm{C}$. Benech de m'avoir confié l'étude des fortifications d'après la carte qu'il a réalisée ; son expertise m'a également permis d'accéder à certains termes techniques et aux dimensions des tracés apparents. La prospection magnétique a été réalisée au moyen d'un gradiomètre à césium, voir ABdul MASsih, BENECH \& Gelin 2009.

43. Pour la commodité de la description, je divise le rempart méridional de la ville basse visible sur la carte géophysique en deux parties de part et d'autre de la première porte, celle à l'ouest étant la muraille occidentale, celle à l'est, la muraille orientale.

44. C'est-à-dire la première porte méridionale connue, par rapport à la seconde située sur le rempart sud actuel.

45. On ne peut cependant l'affirmer sans fouilles, car le secteur prospecté est par trop perturbé pour permettre une lecture nette de l'image obtenue.

46. Dimensions des courtines d'après la carte géophysique. Dans la mesure où certaines limites de murs peuvent apparaître plus ou moins nettes, ces dimensions ne sont pas absolues, mais demeurent cependant de bons indicateurs. 


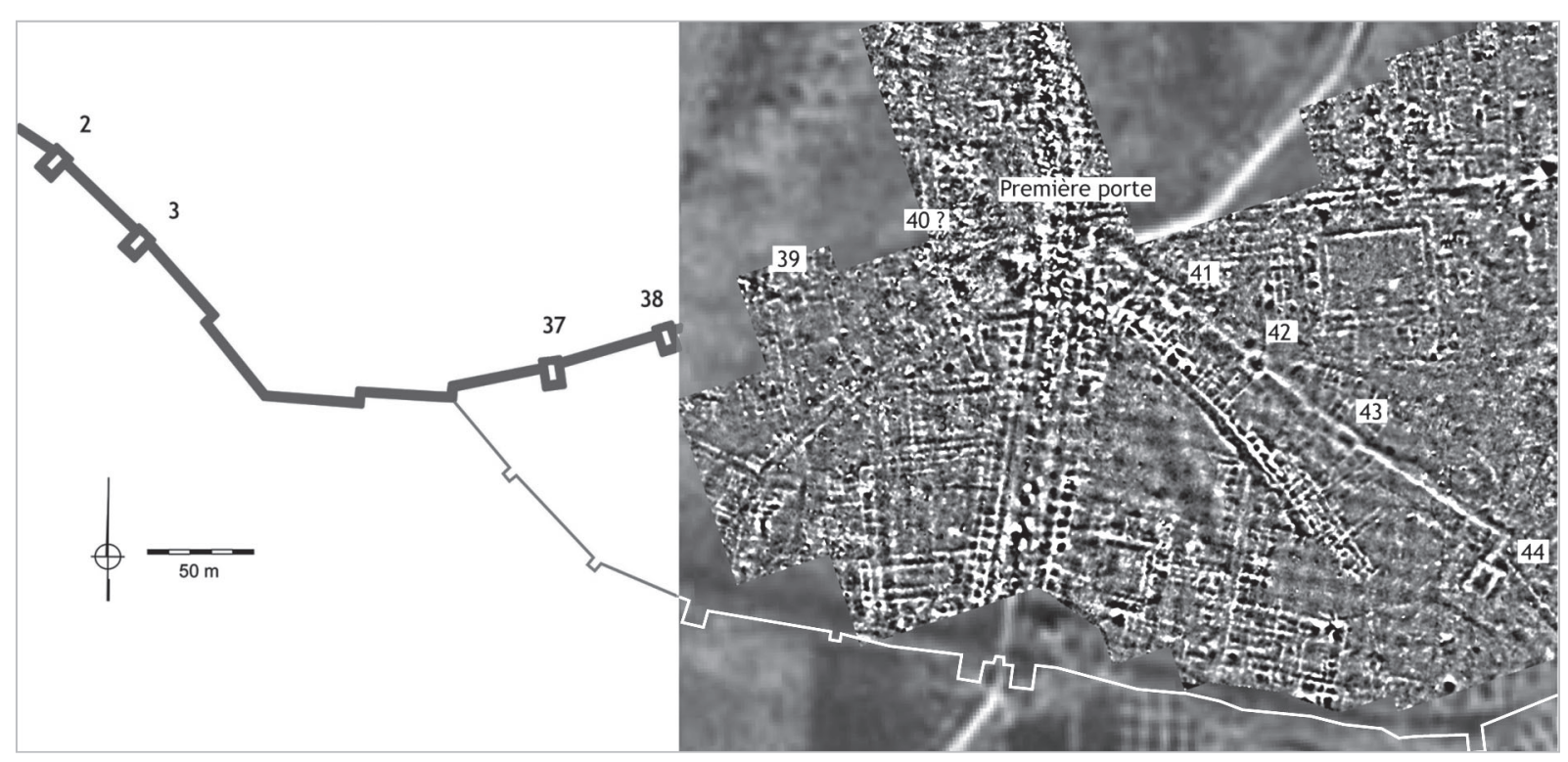

Figure 17. Vue de la partie sud-ouest des fortifications, combinant l'image géophysique et les résultats archéologiques. Le nord est en haut. Plan J. Humbert, S. Baier, S. Knechtel, carte géophysique C. Benech (C) Mission syro-libanaise de Cyrrhus-Nebi Houri.

Les courtines situées aux extrémités du rempart visible sur la carte géophysique de la ville basse sont de dimensions plus importantes. Ainsi, de la tour 38 à la tour 39, la distance est de 59,50 m. L'élément le plus notable cependant est la courtine reliant la tour 43 à la tour 44 : elle est nettement plus longue, $87 \mathrm{~m}$, jusqu'à former un coude à angle aigu vers l'extérieur, qui permet à la muraille de se retourner vers le sud-ouest sur une distance de 15,50 $\mathrm{m}$ avant de joindre la tour 44 .

Quelques anomalies apparaissant sur le tracé des courtines peuvent amener à s'interroger sur l'éventuelle existence de poternes. Ainsi, au centre de la courtine entre la première porte et la tour 41, une zone plus sombre tranchant sur la teinte claire du tracé du rempart pourrait, peut-être, représenter une ouverture. Toujours sur cette courtine, plus près de la tour 41, apparaît une ligne perpendiculaire noire, nette. Enfin, contre la tour 44, à sa jonction avec le rempart, on observe également une ligne noire. D'autres petites anomalies sont visibles, mais il semble difficile de se prononcer en faveur de la trace d'une simple usure, voire destruction de la muraille, ou d'une ouverture.

Dans cette deuxième éventualité, il reste cependant hasardeux d'avancer qu'il s'agit d'ouvertures d'origine car les différentes réoccupations de la ville et, précisément, de la ville basse, rendent impossible, à la seule lecture de la carte géophysique, de déterminer le moment où ces poternes auraient pu être mises en place.

Enfin, à environ une dizaine de mètres à l'extérieur de la ligne des fortifications, il est à noter sur la carte une ligne plus claire ${ }^{47}$ parallèle aux courtines et qui épouse également les tours. Il peut s'agir de l'empreinte d'un fossé ou d'un avant-mur ${ }^{48}$ qui aurait encore renforcé la défense car, dans la ville basse, le faible dénivelé autorisait l'avancée de machines de guerre.

À l'exclusion de la zone de la première porte, relativement perturbée et où il semble difficile d'affirmer la présence de tours à la seule lecture de la carte ${ }^{49}$, on découvre dans la ville basse, où le relief présente

47. Une « anomalie magnétique linéaire ».

48. Un écoulement naturel des eaux de ruissellement le long des murailles n'aurait probablement pas pu créer une ligne aussi régulière.

49. La fouille de la porte, interrompue en 2011, n'avait pas encore atteint les niveaux de tours éventuelles, et seule sa poursuite permettra de résoudre cette question. Par analogie avec les habitudes constructives « grecques », je propose cependant de restituer l'existence de deux tours encadrant la porte, mais cela reste une supposition. 
le plus faible dénivelé, cinq tours (avec la possibilité d'une sixième), toutes à cheval sur le rempart. La tour 39, de $12 \times 11 \mathrm{~m}^{50}$, est située sur l'extrémité orientale de la ligne du rempart descendant de la citadelle, entre la tour 38 (la dernière tour découverte par l'étude archéologique) et la première porte ; c'est entre la tour 39 et la porte que l'on peut probablement intégrer une tour supplémentaire 40, qui prendrait aisément place tout en préservant un espace suffisant pour les courtines.

Puis, sur le rempart oriental, les tours 41 et 42 apparaissent de dimensions assez proches : 14 x $11 \mathrm{~m}$ pour $41,13 \times 11 \mathrm{~m}$ pour 42 , quand la tour 43 mesure $16 \times 13 \mathrm{~m}$. L'ensemble forme des plans plutôt barlongs, alors que ceux des tours situées à la citadelle et sur la pente sont oblongs.

À l'exception de la tour 42 qui empiète de manière claire à l'intérieur de la ville, les tours 39, 41 et 43 semblent moins proéminentes. Cependant, sur la citadelle la première tour 3 ne dépassant pas de plus de 2,50 $\mathrm{m}$ du nu du rempart et les autres tours de la pente n'étant pas différentes, on peut donc considérer que c'est la tour 42 qui présente une particularité, que je n'explique pas ${ }^{51}$.

Enfin la tour 44, sur laquelle je reviendrai plus bas, se distingue plus particulièrement, principalement par ses dimensions (17 $\mathrm{m} \mathrm{E-O} \mathrm{x} \mathrm{13,80} \mathrm{m} \mathrm{N-S)} \mathrm{et} \mathrm{son} \mathrm{emplacement} \mathrm{après} \mathrm{un} \mathrm{coude} \mathrm{et} \mathrm{un} \mathrm{possible} \mathrm{retour}$ de la muraille vers le sud-ouest.

\section{Les courtines et les tours : essai d'interprétation}

Hormis la tour 44, les tours et les courtines de la ville basse sont de dimensions inférieures à celles situées sur la citadelle et la pente. Ceci ne suffit pas, me semble-t-il, à exclure ces deux ensembles de la muraille d'un même mouvement de construction. D'une part, les tours d'autres villes hellénistiques de la région étaient couramment de dimensions proches de celles de Cyrrhus, qu'il s'agisse de la ville basse ou de la citadelle et de la pente ${ }^{52}$, d'autre part le relief environnant et les dispositions des murailles peuvent expliquer ces différences.

Ainsi, si l'on considère le plan général de la muraille méridionale de la citadelle à la ville basse, on constate qu'il peut être divisé en deux grandes parties (fig. 16 et 17) : une première partie comprend toute la zone située depuis la citadelle, dès la tour 1 jusque, dans la pente, à la tour 38 . Sur cette portion des fortifications, le rempart suit un tracé « sortant» : il se projette vers l'extérieur. Cette situation culmine avec le saillant situé entre le décrochement à l'est de la première tour 3 et la tour 37, qui représente le point le plus avancé. Cependant, la forme « sortante » du plan de la muraille a pu constituer un obstacle à la visibilité des défenseurs car, depuis les tours du rempart méridional de la citadelle il devient impossible de voir la ligne du rempart qui court vers l'est dans la pente. De même, depuis la tour 37 et les suivantes dans la pente, les fortifications sud de la citadelle ne peuvent être visibles. Il est probable que ce soit là une raison de l'importance de la projection des tours qui permettait aux défenseurs de se trouver en position dominante laquelle, couplée au dénivelé du relief, offrait un poste d'observation permettant de couvrir angle de vue plus large ${ }^{53}$. Ceci peut également expliquer leur implantation avec de plus grandes distances entre elles. Cette spécificité s'observe notamment à Doura-Europos sur l'Euphrate (fig. 8), où les tours du rempart occidental étaient de plan carré et d'environ $10 \mathrm{~m}$ de côté se projetant à l'extérieur sur plus de $5 \mathrm{~m}$ au-delà du nu du rempart, séparées par des courtines d'environ $60 \mathrm{~m}$ de long; cependant, les tours défendant à la fois les angles nord et sud du rempart et les pentes des wadis attenants, se

50. Dimensions externes des tours d'après la carte géophysique. La première mesure est prise entre les courtines, la deuxième est prise perpendiculairement, depuis l'intérieur de la ville vers l'extérieur.

51. Je ne peux cependant affirmer avec certitude cet aspect, car son tracé est assez peu net, notamment celui de son mur nordest.

52. Parallèles pour la ville basse : tours de $10 \mathrm{~m}$ de côté sur le rempart occidental de Doura-Europos sur l'Euphrate ; pour la citadelle et la pente : 17 x 19 m à Apamée de l'Euphrate (voir GABORIT 2012, p. 201), 17 x 10 m pour les tours d'angle NO et SO de Doura-Europos sur l'Euphrate (voir les relevés de J. Humbert dans les illustrations de Gelin 2000, et VoN GERKAN 1939, fig. 29 vue d'ensemble).

53. Cette situation favorable est également valable pour les décrochements, en dépit de leur projection moindre. 
projetaient de manière plus importante (12 à $15 \mathrm{~m}$ au-delà de la muraille), couvrant un angle de vue plus large et conférant une portée de tir plus conséquente qui pouvait autoriser à la fois une défense supplémentaire des murs et une vue des remparts sud et nord ${ }^{54}$.

La deuxième partie des fortifications méridionales de Cyrrhus se situe entre la tour 38 et la tour 44 : le tracé est « rentrant », le point le plus en retrait étant la première porte, les courtines de part et d'autre formant un entonnoir (un peu large) où des ennemis auraient pu être plus facilement contenus et soumis à des tirs concentrés. Le terrain y est beaucoup moins accidenté ; depuis n'importe quelle tour le regard embrasse l'ensemble des murailles de la ville basse y compris, depuis la partie orientale, celles de la pente jusqu'au saillant. Cependant, là non plus les fortifications sud de la citadelle ne peuvent être vues. Dans la ville basse la taille moindre des tours est compensée par un plus grand nombre de constructions : proportionnellement, elles sont plus nombreuses que sur la pente, où des décrochements pouvaient ponctuer la muraille. Quant à leur plan plutôt barlong, il est possible qu'il ait été imposé par la priorité de surveiller et défendre l'accès à la porte, ainsi que les courtines. Ce dernier aspect semble particulièrement vrai pour la tour 43 , au plan barlong prononcé et associée à la courtine la plus longue de la muraille. On notera également que les deux parties du rempart de la ville basse se croisent presque à angle droit: depuis les tours de chacune des parties, occidentale et orientale, les tirs pouvaient ainsi être dirigés parallèlement au tracé de la muraille attenante, sans risque d'atteindre ses propres défenses sur le côté opposé. Ceci s'entend particulièrement pour les tours 40 et 41 , les plus proches de la première porte et l'une de l'autre.

On peut donc considérer que les dimensions des tours et des courtines sont apparemment associées à la visibilité que les défenseurs pouvaient avoir des fortifications dans leur ensemble.

Les particularités de la longueur de la courtine 43-44 sont évidemment associées à la tour 44, plus grande et avec une projection vers l'extérieur plus importante qui permettait aux défenseurs de couvrir un secteur étendu.

\section{La tour sud-est (44) : essais d'interprétation}

Figure 18. Détail de la tour 44 d'après la carte géophysique. Le nord est en haut. Carte C. Benech (C) Mission syro-libanaise de Cyrrhus-Nebi Houri.

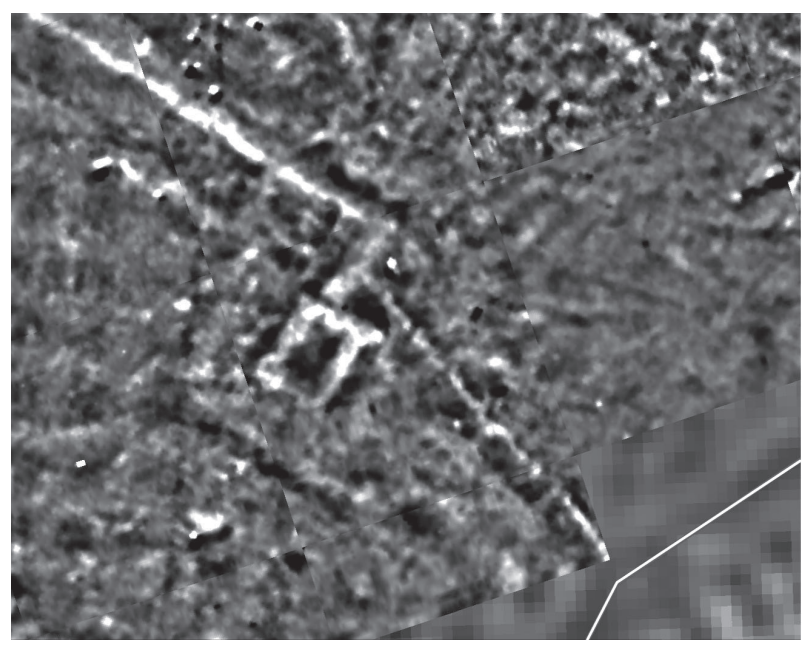

On l'a vu, à l'extrémité sud-est de la ligne des fortifications de la ville basse, se produit une rupture de la ligne de la muraille qui forme un coude à angle presque aigu se retournant vers l'ouest (vers l'extérieur) et qui s'achève avec la tour 44 aux dimensions impressionnantes (fig. 18), dépassant même celles des grandes tours étudiées dans les parties hautes de la ville. Au-delà vers le sud, le rempart

54. Voir les relevés de J. Humbert dans les illustrations de Gelin 2000, et Von Gerkan 1939, notamment la fig. 29 pour une vue d'ensemble. 
en appareil polygonal a disparu ${ }^{55}$ (fig. 16) et n'a, en l'état des recherches, été retrouvé qu'après un deuxième décrochement à l'est de la tour 12 .

Cependant, on devine à l'extérieur de la tour 44 le prolongement du supposé avant-mur (la ligne parallèle au rempart), qui se retourne pour cerner cette grande tour mais prend ensuite une orientation vers l'est à une distance assez éloignée de la tour, puis se retourne à nouveau, cette fois vers le sud-est. Tout se passe comme si ce tracé épousait la forme d'éléments de la muraille qui ont disparu.

Enfin, on peut voir sur la carte géophysique que, parallèlement à cette ligne (dans sa portion sud-est), une ligne plus épaisse, claire, la double à environ $10 \mathrm{~m}$ vers le nord-est. Contre la tour 44 , immédiatement à l'est de son mur oriental, se dessine également une ligne plus claire qui lui est parallèle.

En associant ces différentes observations, le tracé particulier de la courtine 43-44 liée à la tour 44, les grandes dimensions de la tour elle-même et le tracé du supposé avant-mur, plusieurs hypothèses se dessinent :

Dans la première hypothèse (fig. 19 gauche), la ligne parallèle au mur sud-est de la tour 44 représenterait le mur nord-ouest d'une tour identique que l'on peut restituer à proximité immédiate. Ces deux tours pourraient ainsi former une porte d'accès à la ville dont la largeur du passage atteindrait 2 à $3 \mathrm{~m}$; la ligne plus épaisse qui double celle du supposé avant-mur vers le sud-est pourrait représenter un tracé - disparu, probablement arraché partiellement ou totalement - d'une courtine reliant cette supposée deuxième tour au rempart qui se développait au sud-est. Le tracé externe, que je suppose pouvoir être un avant-mur ou un fossé, prend toute sa justification car il englobe ces deux tours et se retourne pour s'aligner le long de la courtine qui prend la direction du rempart méridional. Dans l'hypothèse où il s'agirait d'un mur de protection, l'absence de rupture dans son tracé face au passage de la supposée porte pourrait indiquer, soit l'antériorité des tours et la fermeture du passage au moment de la construction de ce supposé avant-mur, soit la trace de la seule fondation de l'avant-mur et de son ouverture. S'il s'agissait d'un fossé, un passage mobile pouvait aisément se placer et se retirer.

Toujours dans cette hypothèse, on aurait alors une deuxième porte placée sur le rempart de la ville basse, dont l'emplacement contrôlerait à la fois le rempart de la ville basse situé dans la partie rentrante du tracé, et la partie orientale du rempart sud. Cependant, si l'on s'en tient aux tracés proposés, sa
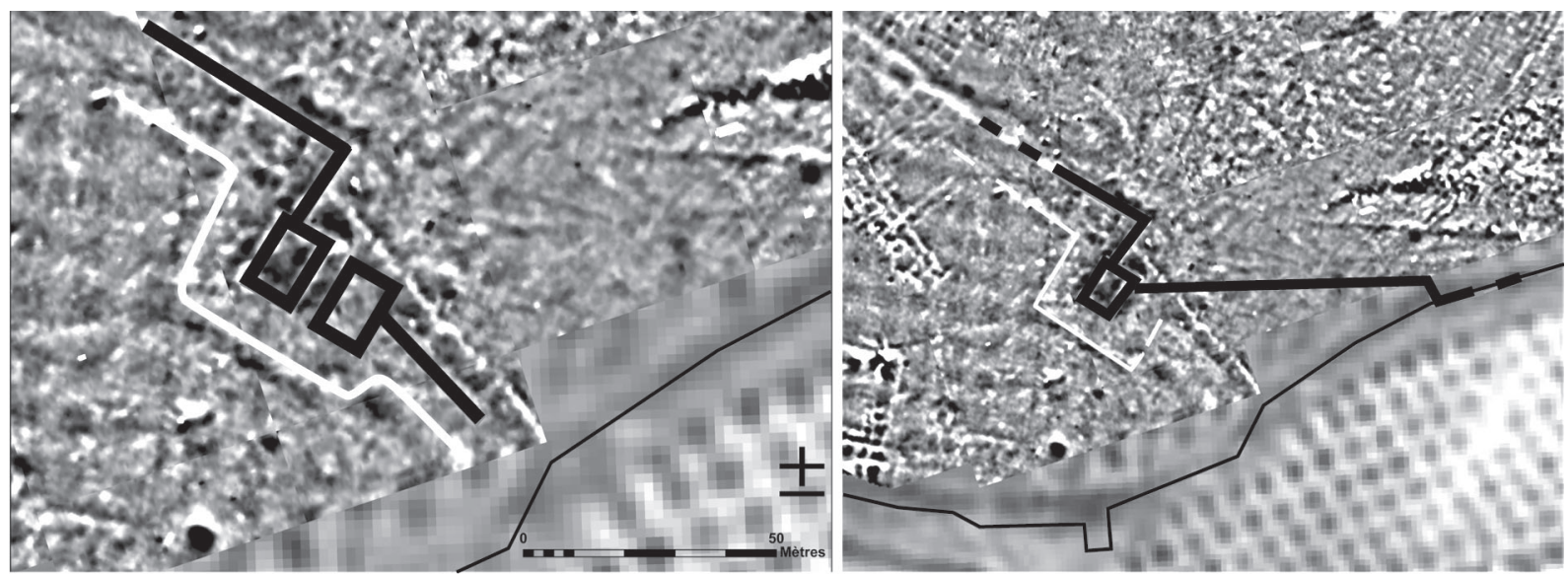

Figure 19. Propositions de restitutions du tracé des murailles attenantes à la tour 44 d'après la carte géophysique (le nord est en haut) : avec une porte près de l'extrémité sud-est (à gauche) ou une tour (à droite). Restitution M. Gelin sur la carte de C. Benech (C) M. Gelin et Mission syro-libanaise de Cyrrhus-Nebi Houri.

55. À l'intérieur de la ville, une ligne blanche fine apparaît, rattachée à la courtine formant le coude, et s'étend vers le sud-est ; je l'interprète comme une construction tardive qui n'appartient pas au système de défense d'origine. 
Figure 20. Proposition de restitution du tracé de la première muraille hellénistique (en noir) de Cyrrhus, suivant l'hypothèse d'un deuxième redan en avancée. En gris, tracé de la muraille méridionale postérieure. Le carroyage est de $150 \mathrm{~m}$. Restitution M. Gelin sur un plan S. Baier, S. Knechtel (C) M. Gelin et Mission syro-libanaise de CyrrhusNebi Houri.

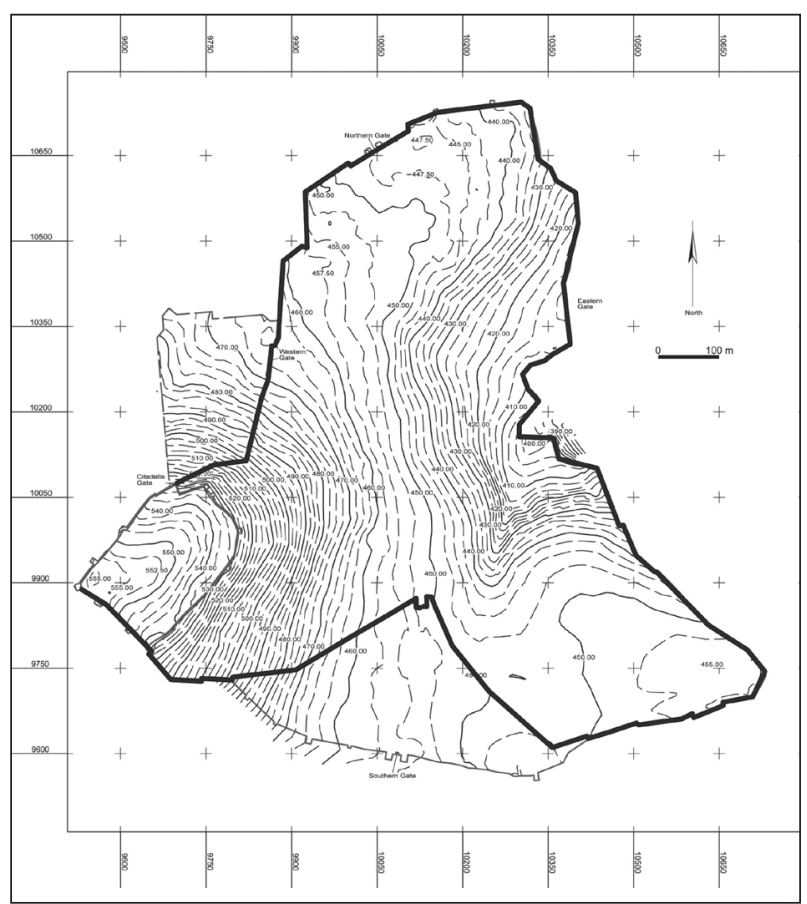

proximité avec la partie orientale du rempart sud aurait formé, à la jonction avec la courtine reliant la tour 44 , un angle saillant qui aurait créé un redan très proche de la tour.

Une deuxième hypothèse (fig. 19 droite) pourrait être celle d'une unique tour de défense avancée, protégeant l'angle formé entre les murailles de la ville basse et celles de la partie sud-est du tracé. Dans ce cas, on doit concevoir un décrochement sur le rempart sud, permettant à cette ligne de se retourner vers le nord-ouest afin de joindre la tour selon un angle évitant tout point mort à la vision depuis la tour.

Enfin, en troisième hypothèse (fig. 20) on peut également supposer que si cette tour n'existait pas dès l'origine, un saillant aurait pu avoir été ménagé à cet emplacement, comme au haut de la pente vers la citadelle. Dans ce cas, comme dans la deuxième hypothèse, on peut restituer un décrochement sur le rempart sud ${ }^{56}$.

Dans tous les cas, porte, tour ou saillant, on aurait là un point de défense se projetant vers l'extérieur et protégeant notamment l'accès au rempart sud et à la première porte de la cité. Il répond, peu ou prou, au saillant existant en haut de la pente, ces deux avancées permettant d'établir un contact visuel entre les défenses de la citadelle et de la ville basse.

En définitive, la carte géophysique permet, très probablement, de restituer un état ancien de la ville basse - que j'associe à la première ville hellénistique connue - qui se caractérise par un système de défense typique des fortifications grecques : des tours à cheval sur le rempart, régulièrement espacées, un avant-mur - ou fossé ? - doublant la ligne des murailles et, au sud-est, une porte, une tour unique ou un saillant protégeant le passage d'accès vers l'intérieur (fig. 19-20).

L'étude de la limite méridionale de la première ville hellénistique de Cyrrhus et de son système défensif a pu opérer un avancement important grâce à la combinaison des études archéologiques et de la prospection géophysique. Les premières ont permis de retrouver des vestiges en place à la citadelle et dans la pente descendant vers la ville basse : socles de courtines et de tours en appareil polygonal

56. Le décrochement entre la tour 44 et la courtine $44-43$ pourrait pérenniser un premier tracé se reliant à un saillant similaire à celui du haut de la pente, mais sa restitution ne m'a pas paru satisfaisante pour la présenter ici. 
supportant une élévation en blocs modulaires de calcaire tendre, ainsi que la porte qui consacre leur tracé. Elles ont également montré que ces constructions représentent la première occupation, qui est d'époque hellénistique, sans pour autant proposer de date plus précise, aucun élément de datation absolue n'ayant été découvert. La carte géophysique offre une vision qui confirme ces recherches, en révélant un tracé de murailles (courtines et tours) qui peut être relié aux vestiges découverts en fouille. Les questions sont nombreuses sur la nature exacte de la tour 44 et leurs réponses ne peuvent se formuler que sous forme d'hypothèses qui sont autant de pistes de travail, que seules de prochaines recherches de terrain pourraient éclairer.

L'ensemble des limites de la première ville peut être restitué ${ }^{57}$ et témoigne d'une construction de fortifications urbaines qui place Cyrrhus dans une tradition défensive typique de l'époque hellénistique en Syrie, et l'associe à des villes fondées par les Diadoques ou leurs successeurs.

\section{BiBLIOGRAPHIE}

Polybe Histoires, Livre V, texte établi et traduit par Paul PÉDECH, Les Belles Lettres, 1977 (Collection des universités de France 255).

Abdul Massin (J.)

$2009 \quad$ «Les mosaïques de la maison romaine et la fortification polygonale de Cyrrhus (Nebi-Houri) - Notes préliminaires " , Syria 86, p. 289-306.

Abdul Massin (J.)

2012 «La mission archéologique libanosyrienne de Cyrrhus, bilan des travaux 2006-2011 », CRAI 2012-1, p. 303-330.

Abdul Massin (J.), Benech (Ch.) \& Gelin (M.)

2009 «First results on the city planning of Cyrrhus (Syria) », Mémoire du sol, espace des hommes, ArchéoSciences 33, suppl., p. 201-203 (http://www.cairn. info/revue-archeosciences-2009-1. htm).

Abdul Massin (J.) \& Gelin (M.)

2010 «Notes préliminaires sur l'étude du système défensif méridional de Cyrrhus. Campagnes 2007-2008», Chronique archéologique en Syrie 4, p. 199-218.

Abdul Massin (J.) \& Gelin (M.)

2016 «Fortifications and town planning in Kyrrhos : its Hellenistic origin and its evolution », FrederiKSEN, SCHNELle, Müth \& SCHNEIDER 2016, p. 207-219.
AL-ShBiB (Sh.)

2012 «Les fortifications de la ville de Cyrrhus. Étude préliminaire », A. Le Bihan, P.-M. Blanc, F. Braemer, J. Dentzer-Feydy \& F. Villeneuve (éd.), Territoires, architecture et matériel au Levant: doctoriales d'archéologie syrienne, Paris-Nanterre 8-9 décembre 2011, Beyrouth (http:// books.openedition.org/ifpo/3012).

AL-Shвів (Sh.)

2015 Les fortifications de Cyrrhus (Nabi Houri), de la période hellénistique à la reconstruction par Justinien, thèse de doctorat, Université Paris 1.

BenECh (C.)

à paraître «Développement urbanistique de la partie sud de Cyrrhus », C. BENECH (éd.), Prospection géophysique et étude des centres urbains de Syrie antique, Lyon.

BESSAC (J.-C.)

1997 La construction des fortifications hellénistiques en pierre de DouraEuropos (Syrie), Thèse d'Histoire, Université de Rennes. 
BESSAC (J.-C.)

2016 «Techniques et économie de la construction des fortifications en pierre: méthodes et perspectives », Frederiksen, Schnelle, Müth \& SCHNEIDER 2016, p. 129-141.

Clarke (G.), McConchie (M.), Reade (W.), Jose-Cougle (L.), O’Hea (M.), Wesselingh (K.), Tidmarsh (J.), Jackson (H.), Grant (K.), Dusting (A.) \& NiXON (C. E. V.)

2011 «Jebel Khalid Report 2009-2010», Mediterranean Archaeology 24, p. 131181.

Desreumaux (A.), Gaborit (J.) \& Caillou (J.-S.)

1999 «Nouvelles découvertes à Apamée d'Osrhoène », CRAIBL, p. 75-105.

Frederiksen (R.), Schnelle (M.), Müth (S.) \& SCHNEIDER (P.) éd.

2016

Focus on Fortifications in the Ancient Mediterranean and the Near East, Fokus Fortifikation Studies 2 (Monographs of the Danish Institute at Athens 18), Oxford.

GABORIT (J.)

2012 La vallée engloutie. Géographie historique du Moyen-Euphrate (BAH 199), Beyrouth, vol. 2.

GeLin (M.)

$2000 \quad$ Histoire et urbanisme d'une ville à travers son architecture de brique crue: l'exemple de Doura-Europos (Syrie orientale hellénistique, parthe et romaine), thèse d'Archéologie des périodes historiques, Université de Paris 1.

GELIN (M.)

2007 «Qalaat el Moudiq, citadelle d'Apamée de l'Oronte. Résultats des travaux de 2004 », J. ABdul MAssiH (éd.), Programme de formation à la préservation du patrimoine culturel syrien (Documents d'Archéologie syrienne 11), Damas, p. 357-382 (version arabe p. 383-399).

GinOuvÈs (R.)

1998 Dictionnaire méthodique de l'architecture grecque et romaine III. Espaces architecturaux, bâtiments et ensembles (Publications de l'École française de Rome 84), Rome.

FrÉzouls (E.)

1977

«Cyrrhus et la Cyrrhestique jusqu'à la fin du Haut-Empire », Aufstieg und Niedergang der römischen Welt II, 8, p. 164-197.

FrÉZOULS (E.)

2012 «Cyrrhus dans l'histoire de la Syrie du Nord », J. Abdul Massin (dir.), Cyrrhus 1. Le théâtre d'après les archives d'Edmond Frézouls (BAH 196), Beyrouth, p. 79-102.

LERICHE (P.)

2000

«Le phénomène urbain dans la Syrie hellénistique », J.-C. DAVID \& M. Al-DbiYat (éd.), La ville en Syrie et ses territoires: héritages et mutations (BEO 52), p. 99-125.

VAN BERCHEM (D.)

1954 «Recherches sur la chronologie des enceintes de Syrie et de Mésopotamie », Syria 31, 3-4, p. 254-270.

VON GERKAN (A.)

1939

« The Fortifications », M. J. RostovtzeFF, F. E. Brown \& C. B. Welles (éd.), The Excavations at Dura-Europos. Preliminary Report of the Seventh and Eighth Seasons of Work, 1933-1934 and 1934-1935, New Haven-Londres, p. 4-62. 